\title{
Ultrarelativistic Spinning Particle and a Rotating Body in External Fields
}

\author{
Alexei A. Deriglazov ${ }^{1,2}$ and Walberto Guzmán Ramírez ${ }^{1}$ \\ ${ }^{1}$ Departamento de Matemática, ICE, Universidade Federal de Juiz de Fora, Juiz de Fora, MG, Brazil \\ ${ }^{2}$ Laboratory of Mathematical Physics, Tomsk Polytechnic University, Lenin Ave. 30, Tomsk 634050, Russia \\ Correspondence should be addressed to Alexei A. Deriglazov; alexei.deriglazov@uff.edu.br
}

Received 14 June 2016; Accepted 17 August 2016

Academic Editor: Seyed H. Hendi

Copyright ( 2016 A. A. Deriglazov and W. G. Ramírez. This is an open access article distributed under the Creative Commons Attribution License, which permits unrestricted use, distribution, and reproduction in any medium, provided the original work is properly cited. The publication of this article was funded by SCOAP ${ }^{3}$.

\begin{abstract}
We use the vector model of spinning particle to analyze the influence of spin-field coupling on the particle's trajectory in ultrarelativistic regime. The Lagrangian with minimal spin-gravity interaction yields the equations equivalent to the Mathisson-PapapetrouTulczyjew-Dixon (MPTD) equations of a rotating body. We show that they have unsatisfactory behavior in the ultrarelativistic limit. In particular, three-dimensional acceleration of the particle becomes infinite in the limit. Therefore, we examine the nonminimal interaction through the gravimagnetic moment $\kappa$ and show that the theory with $\kappa=1$ is free of the problems detected in MPTD equations. Hence, the nonminimally interacting theory seems a more promising candidate for description of a relativistic rotating body in general relativity. Vector model in an arbitrary electromagnetic field leads to generalized Frenkel and BMT equations. If we use the usual special-relativity notions for time and distance, the maximum speed of the particle with anomalous magnetic moment in an electromagnetic field is different from the speed of light. This can be corrected assuming that the three-dimensional geometry should be defined with respect to an effective metric induced by spin-field interaction.
\end{abstract}

\section{Introduction}

The problem of a covariant description of rotational degrees of freedom has a long and fascinating history [1-13]. Equations of motion of a rotating body in curved background were formulated usually in the multipole approach to description of the body; see [1] for the review. The first results were reported by Mathisson [2] and Papapetrou [3]. They assumed that the structure of test body can be described by a set of multipoles and have taken the approximation which involves only first two terms (the pole-dipole approximation). The equations are then derived by integration of conservation law for the energy-momentum tensor, $T^{\mu \nu}{ }_{i \mu}=0$. Manifestly covariant equations were formulated by Tulczyjew [4] and Dixon $[5,6]$. In the current literature, they usually appear in the form given by Dixon (the equations (6.31)-(6.33) in [5]); we will refer to them as Mathisson-Papapetrou-TulczyjewDixon (MPTD) equations. They are widely used now to account for spin effects in compact binaries and rotating black holes; see [14-16] and references therein.
Concerning the equations of spinning particle in electromagnetic field, maybe the best candidates are those of Frenkel $[9,10]$ and Bargmann, Michel, and Telegdi (BMT) [11]. Here, the strong restriction on possible form of semiclassical equations is that the reasonable model should be in correspondence with the Dirac equation. In this regard, the vector model of spin (see below) is of interest because it yields the Frenkel equations at the classical level and implies the Dirac equation after canonical quantization [17].

In this work, we study behavior of a particle governed by these equations (as well as by some of their generalizations) in the ultrarelativistic regime. To avoid the ambiguities in the passage from Lagrangian to Hamiltonian description and vice versa, and in the choice of possible form of interaction, we start in each case from an appropriate variational problem. The vector models of spin provide one possible way to achieve this (for early attempts to build a vector model, see review [18]). In these models, the basic variables in spin sector are $\omega^{\mu}$ and $\pi_{\mu}$, where $\omega^{\mu}$ is non-Grassmann vector and $\pi_{\mu}$ 
represents its conjugated momentum. The spin-tensor is a composite quantity constructed from these variables; $S^{\mu \nu}=$ $2\left(\omega^{\mu} \pi^{\nu}-\omega^{\nu} \pi^{\mu}\right)$. To have a theory with right number of physical degrees of freedom for the spin, certain constraints on the eight basic variables should follow from the variational problem. It should be noted that, even for the free theory in flat space, search for the variational problem represents rather nontrivial task (for the earlier attempts, see [19] and the review [18]).

To explain in a few words the problem which will be under discussion, we recall that typical relativistic equations of motion have singularity at some value of a particle speed. The singularity determines behavior of the particle in ultrarelativistic limit. For instance, the standard equations of spinless particle interacting with electromagnetic field in the physical-time parametrization $x^{\mu}(t)=(c t, \mathbf{x}(t))$,

$$
\frac{d}{d t}\left(\frac{\dot{x}^{\mu}}{\sqrt{-\eta_{\mu \nu} \dot{x}^{\mu} \dot{x}^{\nu}}}\right)=\frac{e}{m c^{2}} F_{\nu}^{\mu} \dot{x}^{\nu}
$$

become singular as the relativistic-contraction factor vanishes, $\eta_{\mu \nu} \dot{x}^{\mu} \dot{x}^{\nu}=c^{2}-\mathbf{v}^{2}=0$. Rewriting the equations in the form of second law of Newton, we find an acceleration. For the case, the longitudinal acceleration reads $a_{\|}=\mathbf{v a}=\left(e\left(c^{2}-\right.\right.$ $\left.\left.\mathbf{v}^{2}\right)^{3 / 2} / m c^{3}\right)(\mathbf{E v})$; that is, the factor, elevated in some degree, appears on the right hand side of the equation and thus determines the value of velocity at which the longitudinal acceleration vanishes, $a_{\|} \stackrel{v \rightarrow c}{\longrightarrow} 0$. For the present case, the singularity implies that, during its evolution in the external field, the spinless particle can not exceed the speed of light $c$.

In the equations for spinning particle, instead of the original metric ( $\eta_{\mu \nu}$ in flat and $g_{\mu \nu}$ in curved space), emerges the effective metric $G_{\mu \nu}=g_{\mu \nu}+h_{\mu \nu}$, with spin- and fielddependent contribution $h_{\mu \nu}$. This turns out to be true for both MPTD and Frenkel equations. This leads to (drastic in some cases) changes $[20,21]$ in behavior of spinning particle as compared with (1). The present work is devoted to detailed analysis of the behavior in ultrarelativistic regime.

We will use the following terminology. The speed $v_{\mathrm{cr}}$ that a particle can not exceed during its evolution in an external field is called critical speed (we prefer the term critical speed instead of maximum speed since $v_{\text {cr }}$ generally is spin- and field-dependent quantity; see below). The observerindependent scale $c$ of special relativity is called, as usual, the speed of light.

The work is organized as follows. In Section 2, we define three-dimensional acceleration (28) of a particle in an arbitrary gravitational field. The definition guarantees that massive spinless particle propagating along four-dimensional geodesic can not exceed the speed of light. Then, we obtain expressions (46) and (47) for the acceleration implied by equation of a general form (45). They will be repeatedly used in the subsequent sections. In Section 3, we shortly review the vector model of spin and present three equivalent Lagrangians of the free theory. In Section 4.1, we obtain equations of the particle minimally interacting with gravity starting from the Lagrangian action without auxiliary variables. The variational problem leads to the theory with fixed value of spin. In Section 4.2, we present the Lagrangian which leads to the model of Hanson-Regge type [22], with unfixed spin and with a mass-spin trajectory constraint. In Section 4.3, we present the MPTD equations in the form convenient for our analysis and show their equivalence with those obtained in Section 4.1. In Section 4.4, we discuss the problems arising in ultrarelativistic limit of MPTD equations. The first problem is the discrepancy between the critical speed and the speed of light. We should note that similar observations were mentioned in a number of works. The appearance of trajectories with space-like four-velocity was remarked by Hanson and Regge in their model of spherical top in electromagnetic field [22]. Space-like trajectories of this model in gravitational fields were studied in $[23,24]$. The second problem is that the transversal acceleration increases with velocity and blows up in the ultrarelativistic limit.

In [25], Khriplovich proposed nonminimal interaction of a rotating body through the gravimagnetic moment $\kappa$. In Section 5.1, we construct the nonminimal interaction starting from the Hamiltonian variational problem and show (Section 5.2) that the model with $\kappa=1$ has reasonable behavior in ultrarelativistic limit. The Lagrangian with one auxiliary variable for the particle with gravimagnetic moment is constructed in Section 5.3. In Section 6 we construct two toy models of spinless particle with critical speed different from the speed of light. In Section 7.1 we analyze generalization of the Frenkel equations to the case of a particle with magnetic moment in an arbitrary electromagnetic field in Minkowski space. Here, we start from the Lagrangian action with one auxiliary variable. In Section 7.2 we show that critical speed of the particle with anomalous magnetic moment is different from the speed of light, if we use the standard special-relativity notions for time and distance. In Section 7.3 we show that the equality between the two speeds can be preserved assuming that three-dimensional geometry should be defined with respect to effective metric arisen due to interaction of spin with electromagnetic field. We point out that a possibility of deformed relation between proper and laboratory time in the presence of electromagnetic field was discussed before by van Holten in his model of spin [26].

Notation. Our variables are taken in arbitrary parametrization $\tau$, and then $\dot{x}^{\mu}=d x^{\mu} / d \tau$. Covariant derivative is $\nabla P^{\mu}=d P^{\mu} / d \tau+\Gamma_{\alpha \beta}^{\mu} \dot{x}^{\alpha} P^{\beta}$ and curvature is $R_{\lambda \mu \nu}^{\sigma}=\partial_{\mu} \Gamma_{\lambda \nu}^{\sigma}-$ $\partial_{\nu} \Gamma_{\lambda \mu}^{\sigma}+\Gamma_{\beta \mu}^{\sigma} \Gamma_{\lambda \nu}^{\beta}-\Gamma_{\beta \nu}^{\sigma} \Gamma_{\lambda \mu}^{\beta}$. The square brackets mean antisymmetrization, $\omega^{[\mu} \pi^{\nu]}=\omega^{\mu} \pi^{\nu}-\omega^{\nu} \pi^{\mu}$. For the fourdimensional quantities, we suppress the contracted indexes and use the notations $\dot{x}^{\mu} G_{\mu \nu} \dot{x}^{\nu}=\dot{x} G \dot{x}, N^{\mu}{ }_{\nu} \dot{x}^{\nu}=(N \dot{x})^{\mu}$, and $\omega^{2}=g_{\mu \nu} \omega^{\mu} \omega^{\nu}, \mu, \nu=0,1,2,3$. Notations for the scalar functions constructed from second-rank tensors are $\theta S=$ $\theta^{\mu \nu} S_{\mu \nu}$ and $S^{2}=S^{\mu \nu} S_{\mu \nu}$.

When we work in four-dimensional Minkowski space with coordinates $x^{\mu}=\left(x^{0}=c t, x^{i}\right)$, we use the metric $\eta_{\mu \nu}=(-,+,+,+)$, then $\dot{x} \omega=\dot{x}^{\mu} \omega_{\mu}=-\dot{x}^{0} \omega^{0}+\dot{x}^{i} \omega^{i}$, and so on. Suppressing the indexes of three-dimensional quantities, we use bold letters: $v^{i} \gamma_{i j} a^{j}=\mathbf{v} \gamma \mathbf{a}, v^{i} G_{i \mu} v^{\mu}=\mathbf{v} G v, i, j=1,2,3$, and so on. 
Electromagnetic field:

$$
\begin{aligned}
F_{\mu \nu}=\partial_{\mu} A_{\nu}-\partial_{\nu} A_{\mu}=\left(F_{0 i}=-E_{i}, \quad F_{i j}=\epsilon_{i j k} B_{k}\right), \\
E_{i}=-\frac{1}{c} \partial_{t} A_{i}+\partial_{i} A_{0}, B_{i}=\frac{1}{2} \epsilon_{i j k} F_{j k}=\epsilon_{i j k} \partial_{j} A_{k} .
\end{aligned}
$$

\section{Three-Dimensional Acceleration of Spinless Particle in General Relativity}

By construction of Lorentz transformations, the speed of light in special relativity is an observer-independent quantity. As we have mentioned in Introduction, the invariant scale is closely related with the critical speed in an external field. In a curved space, we need to be more careful since the three-dimensional geometry should respect the coordinate independence of the speed of light. To achieve this, we use below the Landau and Lifshitz procedure [27] to define time interval, three-dimensional distance, and velocity. Then, we introduce the notion of three-dimensional acceleration which guarantees that massive spinless particle propagating along four-dimensional geodesic can not exceed the speed of light. Expression (47) for longitudinal acceleration implied by equation of the form in (45) will be repeatedly used in subsequent sections.

Consider an observer that labels the events by the coordinates $x^{\mu}$ of pseudo Riemann space $[27,28]$

$$
\mathbf{M}^{(1,3)}=\left\{x^{\mu}, g_{\mu \nu}\left(x^{\rho}\right), \operatorname{sign} g_{\mu \nu}=(-,+,+,+)\right\},
$$

to describe the motion of a particle in gravitational field with metric $g_{\mu v}$. Formal definitions of three-dimensional quantities subject to the discussion can be obtained representing interval in $1+3$ block-diagonal form [27]

$$
\begin{aligned}
-d s^{2}= & g_{\mu \nu} d x^{\mu} d x^{\nu} \\
= & -c^{2}\left[\frac{\sqrt{-g_{00}}}{c}\left(d x^{0}+\frac{g_{0 i}}{g_{00}} d x^{i}\right)\right]^{2} \\
& +\left(g_{i j}-\frac{g_{0 i} g_{0 j}}{g_{00}}\right) d x^{i} d x^{j} .
\end{aligned}
$$

This prompts introducing infinitesimal time interval, distance, and speed as follows:

$$
\begin{aligned}
d t & =\frac{\sqrt{-g_{00}}}{c}\left(d x^{0}+\frac{g_{0 i}}{g_{00}} d x^{i}\right) \equiv-\frac{g_{0 \mu} d x^{\mu}}{c \sqrt{-g_{00}}} . \\
d l^{2} & =\left(g_{i j}-\frac{g_{0 i} g_{0 j}}{g_{00}}\right) d x^{i} d x^{j} \equiv \gamma_{i j} d x^{i} d x^{j}, \\
v & =\frac{d l}{d t} .
\end{aligned}
$$

Therefore, the conversion factor between intervals of the world time $d x^{0} / c$ and the time $d t$ measured by laboratory clock is

$$
\frac{d t}{d x^{0}}=\frac{\sqrt{-g_{00}}}{c}\left(1+\frac{g_{0 i}}{g_{00}} \frac{d x^{i}}{d x^{0}}\right)
$$

Introduce also the three-velocity vector $\mathbf{v}$ with components

$$
v^{i}=\left(\frac{d t}{d x^{0}}\right)^{-1} \frac{d x^{i}}{d x^{0}}
$$

or, symbolically, $v^{i}=d x^{i} / d t$. We stress that, contrary to $d / d x^{\mu}$, the set $\left(d / d t, d / d x^{i}\right)$ is nonholonomic basis of tangent space (let $e_{\mu}=\widetilde{a}_{\mu}^{\alpha} \partial_{\alpha}$ be a basis of tangent space and $e^{\mu}=$ $a^{\mu}{ }_{\alpha} d x^{\alpha}$, where $a^{\mu}{ }_{\alpha} \widetilde{a}^{\alpha}{ }_{\nu}=\delta^{\mu}{ }_{\nu}$, be the dual basis for $e_{\mu}$; i.e., $e^{\mu}\left(e_{\nu}\right)=\delta^{\mu}{ }_{\nu} ; e_{\mu}$ is the holonomic basis (i.e., $e_{\mu}=\partial / \partial x^{\prime \mu}$ is tangent to some coordinate lines $\left.x^{\prime \mu}\right)$ if $\left(e_{\mu} e_{\nu}-e_{\nu} e_{\mu}\right) f=0$; for the matrix $a_{\alpha}^{\mu}$, which determines the dual basis $e^{\mu}$, this condition reduces to the simple equation $\partial_{\mu} a^{\alpha}{ }_{\nu}-\partial_{\nu} a^{\alpha}{ }_{\mu}=0$; for the matrix which determines our $1+3$ decomposition we have $a^{0}{ }_{\mu}=-g_{0 \mu} / c \sqrt{-g_{00}}, a_{0}^{i}=0$, and $a_{j}^{i}=\delta^{i}$; then, for instance, $\partial_{\mu} a^{0}{ }_{\nu}-\partial_{\nu} a_{\mu}^{0}=-\left(1 / c \sqrt{-g_{00}}\right)\left(\partial_{\mu} g_{0 \nu}-\partial_{\nu} g_{0 \mu}\right) \neq 0$; so the set $\left(\partial / \partial t, \partial / \partial x^{i}\right)$ generally does not represent a holonomic basis). This does not represent any special problem for our discussion since we are interested in the differential quantities such as velocity and acceleration.

Equation (8) is consistent with the above definition of $v$ : $v^{2}=(d l / d t)^{2}=\mathbf{v}^{2}=v^{i} \gamma_{i j} v^{j}$. In the result, the interval acquires the form similar to special relativity (but now we have $\mathbf{v}^{2}=\mathbf{v} \gamma \mathbf{v}$ ):

$$
-d s^{2}=-c^{2} d t^{2}+d l^{2}=-c^{2} d t^{2}\left(1-\frac{\mathbf{v}^{2}}{c^{2}}\right) .
$$

This equality holds in any coordinate system $x^{\mu}$. Hence, a particle with the propagation law $d s^{2}=0$ has the speed $\mathbf{v}^{2}=c^{2}$, and this is a coordinate-independent statement.

For the latter use we also introduce the four-dimensional quantity

$$
v^{\mu}=\left(\frac{d t}{d x^{0}}\right)^{-1} \frac{d x^{\mu}}{d x^{0}}=\left(\left(\frac{d t}{d x^{0}}\right)^{-1}, \mathbf{v}\right) .
$$

Combining (8) and (7), we can present the conversion factor in terms of three-velocity as follows:

$$
\left(\frac{d t}{d x^{0}}\right)^{-1}=v^{0}=\frac{c}{\sqrt{-g_{00}}}-\frac{g_{0 i} v^{i}}{g_{00}} .
$$

These rather formal tricks are based [27] on the notion of simultaneity in general relativity and on the analysis of flat limit. Four-interval of special relativity has direct physical interpretation in two cases. First, for two events which occur at the same point, the four-interval is proportional to time interval; $d t=-d s / c$. Second, for simultaneous events, the four-interval coincides with distance; $d l=d s$. Assuming that the same holds in general relativity, let us analyze infinitesimal time interval and distance between two events with coordinates $x^{\mu}$ and $x^{\mu}+d x^{\mu}$. The world line $y^{\mu}=\left(y^{0}, \mathbf{y}=\right.$ const) is associated with laboratory clock placed at the spacial point $\mathbf{y}$. So, the time interval between the events $\left(y^{0}, \mathbf{y}\right)$ and $\left(y^{0}+d y^{0}, \mathbf{y}\right)$ measured by the clock is

$$
d t=-\frac{d s}{c}=\frac{\sqrt{-g_{00}}}{c} d y^{0} .
$$


Consider the event $x^{\mu}$ infinitesimally closed to the world line $\left(y^{0}, \mathbf{y}=\right.$ const $)$. To find the event on the world line which is simultaneous with $x^{\mu}$, we first look for the events $y_{(1)}^{\mu}$ and $y_{(2)}^{\mu}$ which have null-interval with $x^{\mu}, d s\left(x^{\mu}, y_{(a)}^{\mu}\right)=0$. The equation $g_{\mu \nu} d x^{\mu} d x^{\nu}=0$ with $d x^{\mu}=x^{\mu}-y^{\mu}$ has two solutions: $d x_{ \pm}^{0}=g_{0 i} d x^{i} /-g_{00} \pm \sqrt{d \mathbf{x} \gamma d \mathbf{x}} / \sqrt{-g_{00}}$; then $y_{(1)}^{0}=x^{0}-d x_{+}^{0}$ and $y_{(2)}^{0}=x^{0}-d x_{-}^{0}$. Second, we compute the middle point

$$
y^{0}=\frac{1}{2}\left(y_{(1)}^{0}+y_{(2)}^{0}\right)=x^{0}+\frac{g_{0 i} d x^{i}}{g_{00}} .
$$

By definition, the event $\left(y^{0}, \mathbf{y}\right)$ with the null coordinate (13) is simultaneous with the event $\left(x^{0}, \mathbf{x}\right)$ (in the flat limit, the sequence $y_{(1)}^{\mu}, x^{\mu}, y_{(2)}^{\mu}$ of events can be associated with emission, reflection, and absorption of a photon with the propagation law $d s=0$; then the middle point in (13) should be considered simultaneous with $x^{0}$ ). By this way, we synchronized clocks at the spacial points $\mathbf{x}$ and $\mathbf{y}$. According to (13), the simultaneous events have different null-coordinates, and the difference $d x^{0}$ obeys the equation

$$
d x^{0}+\frac{g_{0 i} d x^{i}}{g_{00}}=0
$$

Consider a particle which propagated from $x^{\mu}$ to $x^{\mu}+d x^{\mu}$. Let us compute time interval and distance between these two events. According to (13), the event

$$
\left(x^{0}+d x^{0}+\frac{g_{0 i} d x^{i}}{g_{00}}, \mathbf{x}\right)
$$

at the spacial point $\mathbf{x}$ is simultaneous with $x^{\mu}+d x^{\mu}$.

According to (12) and (13), the time interval between the events $x^{\mu}$ and (15) is

$$
d t=\frac{\sqrt{-g_{00}}}{c}\left(d x^{0}+\frac{g_{0 i}}{g_{00}} d x^{i}\right) .
$$

Since the events $x^{\mu}+d x^{\mu}$ and (15) are simultaneous, this equation gives also the time interval between $x^{\mu}$ and $x^{\mu}+$ $d x^{\mu}$. Further, the difference of coordinates between the events $x^{\mu}+d x^{\mu}$ and (15) is $d z^{\mu}=\left(-g_{0 i} d x^{i} / g_{00}, d x^{i}\right)$. As they are simultaneous, the distance between them is

$$
\begin{aligned}
d l^{2} & =-d s^{2}=g_{\mu \nu} d z^{\mu} d z^{\nu}=\left(g_{i j}-\frac{g_{0 i} g_{0 j}}{g_{00}}\right) d x^{i} d x^{j} \\
& \equiv \gamma_{i j} d x^{i} d x^{j} .
\end{aligned}
$$

Since (15) occurs at the same spacial point as $x^{\mu}$, this equation gives also the distance between $x^{\mu}$ and $x^{\mu}+d x^{\mu}$. Equations (16) and (17) coincide with the formal definitions presented above, in (5) and (6).

We now turn to the definition of three-acceleration. The spinless particle in general relativity follows a geodesic line. If we take the proper time to be the parameter, geodesics obey the system

$$
\begin{aligned}
\nabla_{s} \frac{d x^{\mu}}{d s} & \equiv \frac{d^{2} x^{\mu}}{d s^{2}}+\Gamma_{\alpha \beta}^{\mu} \frac{d x^{\alpha}}{d s} \frac{d x^{\beta}}{d s}=0, \\
g_{\mu \nu} \frac{d x^{\mu}}{d s} \frac{d x^{\nu}}{d s} & =-1,
\end{aligned}
$$

where

$$
\Gamma_{\alpha \beta}^{\mu}=\frac{1}{2} g^{\mu \nu}\left(\partial_{\alpha} g_{\nu \beta}+\partial_{\beta} g_{\alpha \nu}-\partial_{\nu} g_{\alpha \beta}\right)
$$

Due to this definition, system (18) obeys the identity $g_{\mu \nu}\left(d x^{\mu} /\right.$ $d s) \nabla_{s}\left(d x^{\nu} / d s\right)=0$.

The system in this parametrization has no sense of the case we are interested in, $d s^{2} \rightarrow 0$. So, we rewrite it in arbitrary parametrization $\tau$.

$$
\begin{aligned}
& \frac{d \tau}{d s} \frac{d}{d \tau}\left(\frac{d \tau}{d s} \frac{d x^{\mu}}{d \tau}\right)+\left(\frac{d \tau}{d s}\right)^{2} \Gamma_{\alpha \beta}^{\mu}(g) \frac{d x^{\alpha}}{d \tau} \frac{d x^{\beta}}{d \tau}=0, \\
& \frac{d \tau}{d s}=\frac{1}{\sqrt{-\dot{x} g \dot{x}}}
\end{aligned}
$$

this yields the equation of geodesic line in reparametrizationinvariant form

$$
\begin{aligned}
& \frac{1}{\sqrt{-\dot{x} g \dot{x}}} \frac{d}{d \tau}\left(\frac{\dot{x}^{\mu}}{\sqrt{-\dot{x} g \dot{x}}}\right) \\
& =-\Gamma^{\mu}{ }_{\alpha \beta}(g) \frac{\dot{x}^{\alpha}}{\sqrt{-\dot{x} g \dot{x}}} \frac{\dot{x}^{\beta}}{\sqrt{-\dot{x} g \dot{x}}} .
\end{aligned}
$$

Formalism (5)-(9) remains manifestly covariant under subgroup of spacial transformations $x^{0}=x^{\prime 0}, x^{i}=x^{i}\left(x^{\prime j}\right)$, and $\partial x^{i} / \partial x^{\prime j} \equiv a_{j}^{i}\left(x^{\prime}\right)$. Under these transformations, $g_{00}$ is a scalar function and $g_{0 i}$ is a vector while $g_{i j}$ and $\gamma_{i j}$ are tensors. Since $g^{i j} \gamma_{j k}=\delta^{i}{ }_{k}$, the inverse metric of $\gamma_{i j}$ turns out to be $\left(\gamma^{-1}\right)^{i j}=g^{i j}$. Introduce the covariant derivatives $\nabla_{k}$ of a vector field $\xi^{i}\left(x^{0}, x^{k}\right)$ :

$$
\nabla_{k} \xi^{i}=\partial_{k} \xi^{i}+\widetilde{\Gamma}_{k j}^{i}(\gamma) \xi^{j}
$$

The three-dimensional Christoffel symbols $\widetilde{\Gamma}_{j k}^{i}(\gamma)$ are constructed with help of three-dimensional metric $\gamma_{i j}\left(x^{0}, x^{k}\right)$ written in (6), where $x^{0}$ is considered as a parameter:

$$
\widetilde{\Gamma}_{j k}^{i}(\gamma)=\frac{1}{2} \gamma^{i a}\left(\partial_{j} \gamma_{a k}+\partial_{k} \gamma_{a j}-\partial_{a} \gamma_{j k}\right)
$$

As a consequence, the metric $\gamma$ is covariantly constant, $\nabla_{k} \gamma_{i j}=$ 0 .

The velocity in (8) behaves as a vector, $v^{i}\left(x^{0}\right)=$ $a^{i}{ }_{j}\left(x^{\prime k}\left(x^{0}\right)\right) v^{\prime j}\left(x^{0}\right)$, so below we use also the covariant derivative

$$
\nabla_{0} v^{i}=\frac{d v^{i}}{d x^{0}}+\widetilde{\Gamma}_{j k}^{i}(\gamma) \frac{d x^{j}}{d x^{0}} v^{k}
$$


We associated with $\mathbf{M}^{(1,3)}$ the one-parameter family of three-dimensional spaces $\mathbf{M}_{x^{0}}^{3}=\left\{x^{k}, \gamma_{i j}, \nabla_{k} \gamma_{i j}=0\right\}$. Note that velocity has been defined above as a tangent vector to the curve which crosses the family and is parameterized by this parameter, $x^{i}\left(x^{0}\right)$.

To define an acceleration of a particle in the threedimensional geometry, we need the notion of a constant vector field (or, equivalently, the parallel-transport equation). In the case of stationary field, $g_{\mu \nu}\left(x^{k}\right)$, we can identify the curve $x^{i}\left(x^{0}\right)$ of $\mathbf{M}^{(1,3)}$ with that of any one of $\mathbf{M}_{x^{0}}^{3}=\left\{x^{k}, \gamma_{i j}\left(x^{k}\right)\right\}$. So, we have the usual three-dimensional Riemann geometry, and an analog of constant vector field of Euclidean geometry is the covariantly constant field along the line $x^{i}\left(x^{0}\right), \nabla_{0} \xi^{i}=0$. For the field of velocity, its deviation from the covariant constancy is the acceleration [21]

$$
a^{i}=\left(\frac{d t}{d x^{0}}\right)^{-1} \nabla_{0} v^{i}=\left(\frac{d t}{d x^{0}}\right)^{-1} \frac{d v^{i}}{d x^{0}}+\widetilde{\Gamma}^{i}{ }_{j k} v^{j} v^{k} .
$$

To define an acceleration in general case, $\gamma_{i j}\left(x^{0}, x^{i}\right)$, we need to adopt some notion of a constant vector field along the trajectory $x^{i}\left(x^{0}\right)$ that crosses the family $\mathbf{M}_{x^{0}}^{3}$. We propose the definition which preserves one of basic properties of constant fields in differential geometry. In Euclidean and Minkowski spaces, the canonical scalar product of two constant fields does not depend on the point where it was computed. In (pseudo) Riemann space, constant vector field is defined in such a way that the same property holds [29]. In particular, taking the scalar product along a line $x^{i}\left(x^{0}\right)$, we have $\left(d / d x^{0}\right)(\xi, \eta)=0$. For the constant fields in the threedimensional geometry resulting after Landau-Lifshitz $1+3$ decomposition, we demand the same (necessary) condition: $\left(d / d x^{0}\right)\left[\xi^{i}\left(x^{0}\right) \gamma_{i j}\left(x^{0}, x^{i}\left(x^{0}\right)\right) \eta^{i}\left(x^{0}\right)\right]=0$. Taking into account that $\nabla_{k} \gamma_{i j}=0$, this condition can be written as follows:

$$
\left(\nabla_{0} \xi+\frac{1}{2} \xi \partial_{0} \gamma \gamma^{-1}, \eta\right)+\left(\xi, \nabla_{0} \eta+\frac{1}{2} \gamma^{-1} \partial_{0} \gamma \eta\right)=0
$$

This equation is satisfied, if we take the parallel-transport equation to be

$$
\nabla_{0} \xi^{i}+\frac{1}{2}\left(\xi \partial_{0} \gamma \gamma^{-1}\right)^{i}=0
$$

Deviation from the constant field is an acceleration. So we define acceleration with respect to physical time as follows:

$$
a^{i}=\left(\frac{d t}{d x^{0}}\right)^{-1}\left[\nabla_{0} v^{i}+\frac{1}{2}\left(\mathbf{v} \partial_{0} \gamma \gamma^{-1}\right)^{i}\right] .
$$

For the special case of stationary field, $g_{\mu \nu}\left(x^{i}\right)$, definition (28) reduces to (25) and to that of Landau and Lifshitz; see page 251 in [27].

The extra term that appeared in this equation plays an essential role in providing that for the geodesic motion we have $a_{\|} \stackrel{v \rightarrow c}{\longrightarrow} 0$. As a consequence, geodesic particle in gravitational field can not exceed the speed of light. To show this, we compute the longitudinal acceleration ( $\mathbf{v} \gamma \mathbf{a})$ implied by geodesic equation (21). Take $\tau=x^{0}$; then $\sqrt{-\dot{x} g \dot{x}}=(d t /$ $\left.d x^{0}\right) \sqrt{c^{2}-\mathbf{v} \gamma \mathbf{v}}$, and spacial part of (21) is

$$
\left(\frac{d t}{d x^{0}}\right)^{-1} \frac{d}{d x^{0}} \frac{v^{i}}{\sqrt{c^{2}-\mathbf{v} \gamma \mathbf{v}}}=\frac{f^{i}}{\sqrt{c^{2}-\mathbf{v} \gamma \mathbf{v}}},
$$

where

$$
\begin{aligned}
f^{i}\left(v^{\mu}\right)= & -\left(\frac{d t}{d x^{0}}\right)^{-2} \Gamma_{00}^{i}-\Gamma_{j k}^{i} v^{j} v^{k} \\
& -2\left(\frac{d t}{d x^{0}}\right)^{-1} \Gamma_{0 k}^{i} v^{k}=-\Gamma_{\mu \nu}^{i} v^{\mu} v^{\nu}
\end{aligned}
$$

is nonsingular function as $v \rightarrow c$. Computing derivative on the 1.h.s. of (29), we complete $d v^{i} / d x^{0}$ up to covariant derivative $\nabla_{0} v^{i}$ :

$$
\begin{aligned}
\frac{d}{d x^{0}} \frac{v^{i}}{\sqrt{c^{2}-\mathbf{v} \gamma \mathbf{v}}}= & \nabla_{0} v^{i}-\tilde{\Gamma}_{j k}^{i}(\gamma) v^{j} v^{k} \frac{d t}{d x^{0}} \\
& +\frac{v^{i}}{2\left(c^{2}-\mathbf{v} \gamma \mathbf{v}\right)} \frac{d}{d x^{0}}(\mathbf{v} \gamma \mathbf{v}) .
\end{aligned}
$$

For the derivative contained in the last term we find, using covariant constancy of $\gamma$,

$$
\begin{aligned}
\frac{d}{d x^{0}}\left[\mathbf{v} \gamma\left(x^{0}, x^{i}\right) \mathbf{v}\right] & =2 \mathbf{v} \gamma \nabla_{0} \mathbf{v}+\mathbf{v} \partial_{0} \gamma \mathbf{v}+\mathbf{v} \nabla_{0} \gamma \mathbf{v} \\
& =2 \mathbf{v} \gamma \nabla_{0} \mathbf{v}+\mathbf{v} \partial_{0} \gamma \mathbf{v} .
\end{aligned}
$$

Then, (29) acquires the form

$$
\begin{aligned}
& \left(\frac{d t}{d x^{0}}\right)^{-1}\left[M^{i}{ }_{j} \nabla_{0} v^{j}+\frac{\left(\mathbf{v} \partial_{0} \gamma \mathbf{v}\right)}{2\left(c^{2}-\mathbf{v} \gamma \mathbf{v}\right)} v^{i}\right] \\
& =f^{i}+\widetilde{\Gamma}_{k l}^{i} v^{k} v^{l},
\end{aligned}
$$

where

$$
M_{j}^{i}=\delta_{j}^{i}+\frac{v^{i}(\mathbf{v} \gamma)_{j}}{c^{2}-\mathbf{v} \gamma \mathbf{v}} .
$$

We apply the inverse matrix

$$
\widetilde{M}_{j}^{i}=\delta_{j}^{i}-\frac{v^{i}(\mathbf{v} \gamma)_{j}}{c^{2}}
$$

and use the identity

$$
\widetilde{M}_{j}^{i} v^{j}=\frac{c^{2}-\mathbf{v} \gamma \mathbf{v}}{c^{2}} v^{i}
$$

and then

$$
\begin{gathered}
\left(\frac{d t}{d x^{0}}\right)^{-1}\left[\nabla_{0} v^{i}+\frac{\left(\mathbf{v} \partial_{0} \gamma \mathbf{v}\right)}{2 c^{2}} v^{i}\right] \\
=\widetilde{M}^{i}{ }_{j}\left[f^{j}+\widetilde{\Gamma}_{k l}^{j} v^{k} v^{l}\right] .
\end{gathered}
$$


Next, we complete $\nabla_{0} v^{i}$ up to acceleration (28). Then, (37) yields

$$
\begin{aligned}
a^{i}= & \frac{1}{2}\left(\frac{d t}{d x^{0}}\right)^{-1}\left[\left(\mathbf{v} \partial_{0} \gamma \gamma^{-1}\right)^{i}-\frac{\left(\mathbf{v} \partial_{0} \gamma \mathbf{v}\right)}{c^{2}} v^{i}\right] \\
& +\widetilde{M}_{j}^{i}\left[-\Gamma_{\mu \nu}^{j} v^{\mu} v^{\nu}+\widetilde{\Gamma}_{k l}^{j}(\gamma) v^{k} v^{l}\right] .
\end{aligned}
$$

Contracting this with $(\mathbf{v} \gamma)_{i}$, we use $(\mathbf{v} \gamma)_{i} \widetilde{M}_{j}^{i}=\left(\left(c^{2}-\mathbf{v} \gamma \mathbf{v}\right) /\right.$ $\left.c^{2}\right)(\mathbf{v} \gamma)_{j}$ and obtain longitudinal acceleration

$$
\begin{aligned}
\mathbf{v} \gamma \mathbf{a}= & \frac{1}{2}\left(\frac{d t}{d x^{0}}\right)^{-1}\left[\left(\mathbf{v} \partial_{0} \gamma \mathbf{v}\right)-\left(\mathbf{v} \partial_{0} \gamma \mathbf{v}\right) \frac{(\mathbf{v} \gamma \mathbf{v})}{c^{2}}\right] \\
& +\left(1-\frac{\mathbf{v} \gamma \mathbf{v}}{c^{2}}\right)(\mathbf{v} \gamma)_{i}\left[-\Gamma_{\mu \nu}^{i} v^{\mu} v^{\nu}+\widetilde{\Gamma}_{k l}^{i}(\gamma) v^{k} v^{l}\right] .
\end{aligned}
$$

This implies $\mathbf{v} \gamma \mathbf{a} \rightarrow 0$ as $\mathbf{v} \gamma \mathbf{v} \rightarrow c^{2}$.

The last term in (28) yields the important factor $\left(\mathbf{v} \partial_{0} \gamma \mathbf{v}\right)$ in (39). As equations of motion (38) and (39) do not contain the square root $\sqrt{c^{2}-\mathbf{v} \gamma \mathbf{v}}$, they have sense even for $v>c$. Without this factor, we would have $\mathbf{v} \gamma \mathbf{a} \neq 0$ as $\mathbf{v} \gamma \mathbf{v} \rightarrow c^{2}$, so the particle in gravitational field could exceed $c$ and then continue to accelerate. The same happens if we try to define acceleration using usual derivative instead of the covariant one. Indeed, instead of (28), let us define an acceleration according to the expression $a^{i i}=\left(d t / d x^{0}\right)^{-1}\left[d v^{i} / d x^{0}+\right.$ $\left.(1 / 2)\left(v \partial_{0} \gamma \gamma^{-1}\right)^{i}\right]$. Then for the geodesic particle we obtain, instead of (39), the longitudinal acceleration $\mathbf{v} \gamma \mathbf{a}^{\prime}=$ [r.h.s. of (39)] $-(\mathbf{v} \gamma)_{i} \widetilde{\Gamma}^{i}{ }_{j k}(\gamma)\left(d x^{j} / d x^{0}\right) v^{k}=$ [r.h.s. of (39)] $(1 / 2) \partial_{i} \gamma_{j k} v^{i} v^{j} v^{k}$. The extra term does not involve the factor $c^{2}-\mathbf{v} \gamma \mathbf{v}$ and so does not vanish at $|v|=c$.

Let us confirm that $c$ is the only special point of function (39) representing the longitudinal acceleration. Using (19), (6)-(10), (23), and the identities

$$
\begin{aligned}
& \gamma_{i j} g^{j k}=\delta_{i}^{k}, \\
& \gamma_{i j} g^{j 0}=-\frac{g_{0 i}}{g_{00}},
\end{aligned}
$$

we can present the right hand side of (39) in terms of initial metric as follows:

$$
\begin{aligned}
& \mathbf{v} \gamma \mathbf{a} \\
& =\frac{c^{2}-\mathbf{v} \gamma \mathbf{v}}{2 c \sqrt{-g_{00}}}\left\{\frac{c}{\sqrt{-g_{00}}}\left[\left(\frac{d t}{d x^{0}}\right)^{-1} \partial_{0} g_{00}+v^{k} \partial_{k} g_{00}\right]\right. \\
& -\partial_{0} g_{00}\left(\frac{d t}{d x^{0}}\right)^{-2}-2 \partial_{0} g_{0 k}\left(\frac{d t}{d x^{0}}\right)^{-1} v^{k} \\
& \left.-\partial_{0} g_{k l} v^{k} v^{l}\right\} \equiv \frac{c^{2}-\mathbf{v} \gamma \mathbf{v}}{2 c \sqrt{-g_{00}}}\left\{\frac{c}{\sqrt{-g_{00}}} v^{\mu} \partial_{\mu} g_{00}\right. \\
& \left.-\partial_{0} g_{\mu \nu} v^{\mu} v^{\nu}\right\} .
\end{aligned}
$$

The quantity $v^{\mu}$ has been defined in (10). Excluding $v^{0}$ according to this expression, we obtain

$$
\begin{aligned}
\mathbf{v} \gamma \mathbf{a} & =\frac{c^{2}-\mathbf{v} \gamma \mathbf{v}}{2 \sqrt{-g_{00}}}\left\{\frac{v^{k} \partial_{k} g_{00}}{\sqrt{-g_{00}}}-2 \partial_{0}\left(\frac{g_{0 i}}{\sqrt{-g_{00}}}\right) v^{i}\right. \\
- & \left.\frac{1}{c} \partial_{0} \gamma_{i j} v^{i} v^{j}\right\} .
\end{aligned}
$$

For the stationary metric, $g_{\mu \nu}\left(x^{k}\right),(42)$ acquires a specially simple form:

$$
\mathbf{v} \gamma \mathbf{a}=-\left(c^{2}-\mathbf{v} \gamma \mathbf{v}\right) \frac{v^{k} \partial_{k} g_{00}}{2 g_{00}} .
$$

This shows that the longitudinal acceleration has only one special point in the stationary gravitational field; $\mathbf{v} \gamma \mathbf{a} \rightarrow 0$ as $\mathbf{v} \gamma \mathbf{v} \rightarrow c^{2}$. Then, the same is true in general case (41), at least for the metric which is sufficiently slowly varied in time.

While we have discussed the geodesic equation, the computation which leads to formula (39) can be repeated for a more general equation. Let us formulate the result which will be repeatedly used below. Using the factor $\sqrt{-\dot{x} g \dot{x}}$, we construct the reparametrization-invariant derivative

$$
D=\frac{1}{\sqrt{-\dot{x} g \dot{x}}} \frac{d}{d \tau} .
$$

Consider the reparametrization-invariant equation of the form

$$
D D x^{\mu}(\tau)=\mathscr{F}^{\mu}\left(D x^{\nu}, \ldots\right)
$$

and suppose that the three-dimensional geometry is defined by $g_{\mu \nu}$. Then, (45) implies the three-acceleration

$$
\begin{aligned}
a^{i}= & \widetilde{M}_{j}^{i}\left[\left(c^{2}-\mathbf{v} \gamma \mathbf{v}\right) \mathscr{F}^{j}+\widetilde{\Gamma}_{k l}^{j}(\gamma) v^{k} v^{l}\right] \\
& +\frac{1}{2}\left(\frac{d t}{d x^{0}}\right)^{-1}\left[\left(\mathbf{v} \partial_{0} \gamma \gamma^{-1}\right)^{i}-\frac{v^{i}}{c^{2}}\left(\mathbf{v} \partial_{0} \gamma \mathbf{v}\right)\right]
\end{aligned}
$$

and the longitudinal acceleration

$$
\begin{aligned}
\mathbf{v} \gamma \mathbf{a} & =\frac{\left(c^{2}-\mathbf{v} \gamma \mathbf{v}\right)^{2}}{c^{2}}(\mathbf{v} \gamma \mathscr{F}) \\
+ & \frac{c^{2}-\mathbf{v} \gamma \mathbf{v}}{c^{2}}\left[(\mathbf{v} \gamma)_{i} \widetilde{\Gamma}_{k l}^{i}(\gamma) v^{k} v^{l}\right. \\
& \left.+\frac{1}{2}\left(\frac{d t}{d x^{0}}\right)^{-1}\left(\mathbf{v} \partial_{0} \gamma \mathbf{v}\right)\right] .
\end{aligned}
$$

The spacial part of the force is $\mathscr{F}^{i}=\mathscr{F}^{i}\left(v^{\nu} / \sqrt{c^{2}-\mathbf{v} \gamma \mathbf{v}}\right)$, where $v^{\mu}$ is given by (10) and the connection $\widetilde{\Gamma}_{k l}^{i}(\gamma)$ is constructed with help of the three-dimensional metric $\gamma_{i j}=\left(g_{i j}-\right.$ $\left.g_{0 i} g_{0 j} / g_{00}\right)$ according to (23). For the geodesic equation in this notation, we have $\mathscr{F}^{i}=-\Gamma_{\mu \nu}^{i}\left(v^{\mu} v^{\nu} /\left(c^{2}-\mathbf{v} \gamma \mathbf{v}\right)\right)$. With this $\mathscr{F}^{i},(46)$ and (47) coincide with (38) and (39). 


\section{Vector Model of Relativistic Spin}

The variational problem for vector model of spin interacting with electromagnetic and gravitational fields can be formulated with various sets of auxiliary variables [17, 30-33].
For the free theory in flat space, there is Lagrangian action without auxiliary variables. Configuration space consists of the position $x^{\mu}(\tau)$ and non-Grassmann vector $\omega^{\mu}(\tau)$ attached to the point $x^{\mu}$. The action reads [20,32]

$$
S=-\frac{1}{\sqrt{2}} \int d \tau \sqrt{m^{2} c^{2}-\frac{\alpha}{\omega^{2}}} \sqrt{-\dot{x} N \dot{x}-\dot{\omega} N \dot{\omega}+\sqrt{[\dot{x} N \dot{x}+\dot{\omega} N \dot{\omega}]^{2}-4(\dot{x} N \dot{\omega})^{2}}}
$$

The matrix $N_{\mu \nu}$ is the projector on the plane orthogonal to $\omega^{\nu}$ :

$$
\begin{aligned}
N_{\mu \nu} & =\eta_{\mu \nu}-\frac{\omega_{\mu} \omega_{\nu}}{\omega^{2}}, \\
\text { then } N_{\mu \alpha} N^{\alpha \nu} & =N_{\mu}{ }^{\nu}, \\
N_{\mu \nu} \omega^{\nu} & =0 .
\end{aligned}
$$

The double square-root structure in expression (48) seems to be typical for the vector models of spin [22, 34]. This yields the primary constraint $T_{4}$ in (62) and, at the end, supplementary spin condition (77). The parameter $m$ is mass, while $\alpha$ determines the value of spin. The value $\alpha=3 \hbar^{2} / 4$ corresponds to an elementary spin one-half particle. The model is invariant under reparametrizations and local spinplane symmetries [35] (the reparametrizations are $\tau \rightarrow \tau^{\prime}(\tau)$, $x^{\prime \mu}\left(\tau^{\prime}\right)=x^{\mu}(\tau)$, and $\omega^{\prime \mu}\left(\tau^{\prime}\right)=\omega^{\mu}(\tau)$; i.e., both $x$ and $\omega$ are scalar functions; the local spin-plane transformations act in the plane determined by the vectors $\omega^{\mu}$ and $\pi^{\nu}$ ).

The spin is described by Frenkel spin-tensor [9]. In our model, this is a composite quantity constructed from $\omega^{\mu}$ and its conjugated momentum $\pi_{\mu}=\partial L / \partial \dot{\omega}^{\mu}$ as follows:

$$
S^{\mu \nu}=2\left(\omega^{\mu} \pi^{\nu}-\omega^{\nu} \pi^{\mu}\right)=\left(S^{i 0}=D^{i}, S_{i j}=2 \epsilon_{i j k} S_{k}\right),
$$

and then $S_{i}=\epsilon_{i j k} \omega_{j} \pi_{k}=(1 / 4) \epsilon_{i j k} S_{j k}$. Here, $S_{i}$ is threedimensional spin-vector and $D_{i}$ is dipole electric moment [12]. In contrast to its constituents $\omega^{\mu}$ and $\pi^{\nu}$, the spintensor is invariant under local spin-plane symmetry and thus represents an observable quantity. Canonical quantization of the model yields one-particle sector of the Dirac equation [17].

In formulation (48), the model admits minimal interaction with electromagnetic field and with gravity. This does not spoil the number and the algebraic structure of constraints presented in the free theory. To describe the spinning particle with magnetic and gravimagnetic moments, we will need the following two reformulations.

In the spinless limit, $\alpha=0$ and $\omega^{\mu}=0$, functional (48) reduces to the standard expression, $-m c \sqrt{-\dot{x}^{\mu} \dot{x}_{\mu}}$. The latter can be written in equivalent form using the auxiliary variable $\lambda(\tau)$ as follows: $(1 / 2 \lambda) \dot{x}^{2}-(\lambda / 2) m^{2} c^{2}$. Similarly to this, (48) can be presented in the equivalent form

$$
\begin{aligned}
L & =\frac{1}{4 \lambda_{1}}[\dot{x} N \dot{x}+\dot{\omega} N \dot{\omega} \\
& \left.-\sqrt{[\dot{x} N \dot{x}+\dot{\omega} N \dot{\omega}]^{2}-4(\dot{x} N \dot{\omega})^{2}}\right]-\frac{\lambda_{1}}{2}\left[(m c)^{2}\right. \\
& \left.-\frac{\alpha}{\omega^{2}}\right] .
\end{aligned}
$$

In this formulation, our model admits interaction of spin with an arbitrary electromagnetic field through the magnetic moment; see Section 7.1. Another form of the Lagrangian is

$$
\begin{gathered}
L=-\sqrt{(m c)^{2}-\frac{\alpha}{\omega^{2}}} \sqrt{\left(1-\lambda^{2}\right)^{-1}[-\dot{x} N \dot{x}-\dot{\omega} N \dot{\omega}+2 \lambda \dot{x} N \dot{\omega}]} \equiv \\
-\sqrt{(m c)^{2}-\frac{\alpha}{\omega^{2}}} \sqrt{-(N \dot{x}, N \dot{\omega})\left(\begin{array}{cc}
\frac{\eta}{1-\lambda^{2}} & \frac{-\lambda \eta}{1-\lambda^{2}} \\
\frac{-\lambda \eta}{1-\lambda^{2}} & \frac{\eta}{1-\lambda^{2}}
\end{array}\right)\left(\begin{array}{c}
N \dot{x} \\
N \dot{\omega}
\end{array}\right) .}
\end{gathered}
$$

Its advantage is that the expression under the square root represents quadratic form with respect to the velocities $\dot{x}$ and $\dot{\omega}$. To relate Lagrangians (48) and (52), we exclude $\lambda$ from the latter. Computing variation of (52) with respect to $\lambda$, we obtain the equation

$$
(\dot{x} N \dot{\omega}) \lambda^{2}-(\dot{x} N \dot{x}+\dot{\omega} N \dot{\omega}) \lambda+(\dot{x} N \dot{\omega})=0,
$$

which determines $\lambda$ :

$$
\begin{aligned}
& \lambda_{ \pm} \\
& =\frac{(\dot{x} N \dot{x}+\dot{\omega} N \dot{\omega}) \pm \sqrt{(\dot{x} N \dot{x}+\dot{\omega} N \dot{\omega})^{2}-4(\dot{x} N \dot{\omega})^{2}}}{2(\dot{x} N \dot{\omega})} .
\end{aligned}
$$

We substitute $\lambda_{+}$into (52) and use $\lambda_{+} \lambda_{-}=1$, and then (52) turns into (48). In formulation (52), our model admits interaction of spin with gravity through the gravimagnetic moment; see Section 5.3.

\section{Minimal Interaction with an Arbitrary Gravitational Field}

4.1. Lagrangian and Hamiltonian Formulations. The minimal interaction with gravitational field can be achieved by covariantization of the formulation without auxiliary variables. In 
expressions (48) and (49), we replace $\eta_{\mu \nu} \rightarrow g_{\mu \nu}$ and usual derivative by the covariant one; $\dot{\omega}^{\mu} \rightarrow \nabla \omega^{\mu}=d \omega^{\mu} / d \tau+$ $\Gamma_{\alpha \beta}^{\mu} \dot{x}^{\alpha} \omega^{\beta}$. Thus, our Lagrangian in a curved background reads [33]

$$
\begin{aligned}
L & =-\frac{1}{\sqrt{2}}\left[m^{2} c^{2}-\frac{\alpha}{\omega^{2}}\right]^{1 / 2} \\
& \cdot \sqrt{-\dot{x} N \dot{x}-\nabla \omega N \nabla \omega+\sqrt{[\dot{x} N \dot{x}+\nabla \omega N \nabla \omega]^{2}-4(\dot{x} N \nabla \omega)^{2}}} \\
& \equiv-\frac{1}{\sqrt{2}}\left[m^{2} c^{2}-\frac{\alpha}{\omega^{2}}\right]^{1 / 2} L_{0} .
\end{aligned}
$$

Velocities $\dot{x}^{\mu}, \nabla \omega^{\mu}$ and projector $N_{\mu \nu}$ transform like contravariant vectors and covariant tensor, so the action is manifestly invariant under general-coordinate transformations.

Let us construct Hamiltonian formulation of model (56). Conjugate momenta for $x^{\mu}$ and $\omega^{\mu}$ are $p_{\mu}=\partial L / \partial \dot{x}^{\mu}$ and $\pi_{\mu}=\partial L / \partial \dot{\omega}^{\mu}$, respectively. Due to the presence of Christoffel symbols in $\nabla \omega^{\mu}$, the conjugated momentum $p_{\mu}$ does not transform as a vector, so it is convenient to introduce the canonical momentum

$$
P_{\mu} \equiv p_{\mu}-\Gamma_{\alpha \mu}^{\beta} \omega^{\alpha} \pi_{\beta}
$$

the latter transforms as a vector under general transformations of coordinates. Manifest form of the momenta is as follows:

$$
\begin{aligned}
& P_{\mu}=\frac{1}{\sqrt{2} L_{0}}\left[m^{2} c^{2}-\frac{\alpha}{\omega^{2}}\right]^{1 / 2}\left[N_{\mu \nu} \dot{x}^{\nu}-K_{\mu}\right], \\
& \pi_{\mu}=\frac{1}{\sqrt{2} L_{0}}\left[m^{2} c^{2}-\frac{\alpha}{\omega^{2}}\right]^{1 / 2}\left[N_{\mu \nu} \nabla \omega^{\nu}-R_{\mu}\right],
\end{aligned}
$$

with

$$
\begin{aligned}
K_{\mu} & =T^{-1 / 2}\left[(\dot{x} N \dot{x}+\nabla \omega N \nabla \omega)(N \dot{x})_{\mu}\right. \\
& \left.-2(\dot{x} N \nabla \omega)(N \nabla \omega)_{\mu}\right], \\
R_{\mu} & =T^{-1 / 2}\left[(\dot{x} N \dot{x}+\nabla \omega N \nabla \omega)(N \nabla \omega)_{\mu}\right. \\
& \left.-2(\dot{x} N \nabla \omega)(N \dot{x})_{\mu}\right] .
\end{aligned}
$$

These vectors obey the following remarkable identities:

$$
\begin{aligned}
K^{2} & =\dot{x} N \dot{x}, \\
R^{2} & =\nabla \omega N \nabla \omega, \\
K R & =-\dot{x} N \nabla \omega, \\
\dot{x} R+\nabla \omega K & =0 \\
K \dot{x}+R \nabla \omega & =\sqrt{[\dot{x} N \dot{x}+\nabla \omega N \nabla \omega]^{2}-4(\dot{x} N \nabla \omega)^{2}} .
\end{aligned}
$$

Using (49), we conclude that $\omega \pi=0$ and $P \omega=0$; that is, we found two primary constraints. Using the relations in (60), we find one more primary constraint, $P \pi=0$. At last, computing
$P^{2}+\pi^{2}$ given by (58); we see that all the terms with derivatives vanish, and we obtain the primary constraint

$$
T_{1} \equiv P^{2}+m^{2} c^{2}+\pi^{2}-\frac{\alpha}{\omega^{2}}=0
$$

In the result, action (56) implies four primary constraints, $T_{1}$ and

$$
\begin{aligned}
& T_{2} \equiv \omega \pi=0, \\
& T_{3} \equiv P \omega=0, \\
& T_{4} \equiv P \pi=0 .
\end{aligned}
$$

The Hamiltonian is constructed excluding velocities from the expression

$$
H=p_{\mu} \dot{x}+\pi \dot{\omega}-L+\lambda_{i} T_{i} \equiv P \dot{x}+\pi \nabla \omega-L+\lambda_{i} T_{i},
$$

where $\lambda_{i}$ is the Lagrangian multipliers associated with the primary constraints. From (58), we observe the equalities $P \dot{x}=\left(\sqrt{2} L_{0}\right)^{-1}\left(m^{2} c^{2}-\alpha / \omega^{2}\right)^{1 / 2}[\dot{x} N \dot{x}-\dot{x} K]$ and $\pi \nabla \omega=$ $\left(\sqrt{2} L_{0}\right)^{-1}\left(m^{2} c^{2}-\alpha / \omega^{2}\right)^{1 / 2}[\nabla \omega N \nabla \omega-\nabla \omega R]$. Together with (60), they imply $P \dot{x}+\pi \nabla \omega=L$. Using this in (63), we conclude that the Hamiltonian is composed of the primary constraints

$$
\begin{aligned}
H= & \frac{\lambda_{1}}{2}\left(P^{2}+m^{2} c^{2}+\pi^{2}-\frac{\alpha}{\omega^{2}}\right)+\lambda_{2}(\omega \pi) \\
& +\lambda_{3}(P \omega)+\lambda_{4}(P \pi) .
\end{aligned}
$$

The full set of phase-space coordinates consists of the pairs $x^{\mu}, p_{\mu}$ and $\omega^{\mu}, \pi_{\mu}$. They fulfill the fundamental Poisson brackets $\left\{x^{\mu}, p_{\nu}\right\}=\delta_{\nu}^{\mu}$ and $\left\{\omega^{\mu}, \pi_{\nu}\right\}=\delta_{\nu}^{\mu}$ and then $\left\{P_{\mu}, P_{\nu}\right\}=$ $R_{\lambda \mu \nu}^{\sigma} \pi_{\sigma} \omega^{\lambda},\left\{P_{\mu}, \omega^{\nu}\right\}=\Gamma_{\mu \alpha}^{\nu} \omega^{\alpha}$, and $\left\{P_{\mu}, \pi_{\nu}\right\}=-\Gamma_{\mu \nu}^{\alpha} \pi_{\alpha}$. For the quantities $x^{\mu}, P^{\mu}$, and $S^{\mu \nu}$, these brackets imply the typical relations used by people for spinning particles in Hamiltonian formalism.

$$
\begin{aligned}
\left\{x^{\mu}, P_{\nu}\right\} & =\delta_{\nu}^{\mu}, \\
\left\{P_{\mu}, P_{\nu}\right\} & =-\frac{1}{4} R_{\mu \nu \alpha \beta} S^{\alpha \beta}, \\
\left\{P_{\mu}, S^{\alpha \beta}\right\} & =\Gamma_{\mu \sigma}^{\alpha} S^{\sigma \beta}-\Gamma_{\mu \sigma}^{\beta} S^{\sigma \alpha}, \\
\left\{S^{\mu \nu}, S^{\alpha \beta}\right\} & =2\left(g^{\mu \alpha} S^{\nu \beta}-g^{\mu \beta} S^{\nu \alpha}-g^{\nu \alpha} S^{\mu \beta}+g^{\nu \beta} S^{\mu \alpha}\right) .
\end{aligned}
$$

To reveal the higher-stage constraints and the Lagrangian multipliers, we study the equation $\dot{T}_{i}=\left\{T_{i}, H\right\}=0 . T_{2}$ implies the secondary constraint

$$
\dot{T}_{2}=0 \Longrightarrow T_{5} \equiv \pi^{2}-\frac{\alpha}{\omega^{2}} \approx 0 ;
$$

then $T_{1}$ can be replaced on $P^{2}+m^{2} c^{2} \approx 0$. Preservation in time of $T_{4}$ and $T_{3}$ gives the Lagrangian multipliers $\lambda_{3}$ and $\lambda_{4}$ :

$$
\begin{aligned}
& \lambda_{3}=2 a \lambda_{1}(\pi \theta P), \\
& \lambda_{4}=-2 a \lambda_{1}(\omega \theta P),
\end{aligned}
$$

where we have denoted

$$
\begin{aligned}
\theta_{\mu \nu} & \equiv R_{\alpha \beta \mu \nu} S^{\alpha \beta}, \\
a & =\frac{2}{16 m^{2} c^{2}+(\theta S)} .
\end{aligned}
$$


TABLE 1: Algebra of constraints.

\begin{tabular}{lccccc}
\hline & $T_{1}$ & $T_{5}$ & $T_{2}$ & $T_{3}$ & $T_{4}$ \\
\hline$T_{1}=P^{2}+m^{2} c^{2}$ & 0 & 0 & 0 & $\frac{1}{2}(\omega \theta P)$ & $\frac{1}{2}(\pi \theta P)$ \\
$T_{5}=\pi^{2}-\frac{\alpha}{\omega^{2}}$ & 0 & 0 & $-2 T_{5}$ & $-2 T_{4}$ & $-\frac{2 T_{3}}{\omega^{2}}$ \\
$T_{2}=\omega \pi$ & 0 & $2 T_{5}$ & 0 & $-T_{3}$ & $T_{4}$ \\
$T_{3}=P \omega$ & $-\frac{1}{2}(\omega \theta P)$ & $2 T_{4}$ & $T_{3}$ & 0 & $-\frac{1}{8 a}$ \\
$T_{4}=P \pi$ & $-\frac{1}{2}(\pi \theta P)$ & $\frac{2 T_{3}}{\omega^{2}}$ & $-T_{4}$ & $\frac{1}{8 a}$ & 0 \\
\hline
\end{tabular}

Preservation in time of $T_{1}$ gives the equation $\lambda_{3}(\omega \theta P)+$ $\lambda_{4}(\pi \theta P)=0$ which is identically satisfied by virtue of $(67)$. No more constraints are generated after this step. We summarize the algebra of Poisson brackets between the constraints in Table $1 . T_{3}$ and $T_{4}$ represent a pair of second-class constraints, while $T_{2}, T_{5}$, and the combination

$$
T_{0}=T_{1}+4 a(\pi \theta P) T_{3}-4 a(\omega \theta P) T_{4}
$$

are the first-class constraints. Taking into account that each second-class constraint rules out one phase-space variable, whereas each first-class constraint rules out two variables, we have the right number of spin degrees of freedom, $8-(2+4)=$ 2.

It should be noted that $\omega^{\mu}$ and $\pi^{\mu}$ turn out to be spacelike vectors. Indeed, in flat limit and in the frame where $p^{\mu}=$ $\left(p^{0}, \mathbf{0}\right)$, the constraints $\omega p=\pi p=0$ imply $\omega^{0}=\pi^{0}=0$. This implies $\omega^{2} \geq 0$ and $\pi^{2} \geq 0$. Combining this with constraint (66), we conclude $\omega^{2}>0$ and $\pi^{2}>0$.

We point out that the first-class constraint $T_{5}=\pi^{2}-$ $\alpha / \omega^{2} \approx 0$ can be replaced on the pair

$$
\begin{aligned}
& \pi^{2}=\text { const }, \\
& \omega^{2}=\text { const } ;
\end{aligned}
$$

this gives an equivalent formulation of the model. The Lagrangian which implies constraints (62) and (71) has been studied in [17, 30,31]. Hamiltonian and Lagrangian equations for physical variables of the two formulations coincide [32], which proves their equivalence.

Using (67), we can present Hamiltonian (64) in the form

$H$

$$
\begin{aligned}
= & \frac{\lambda_{1}}{2}\left(P^{2}+m^{2} c^{2}+4 a[(\pi \theta P)(P \omega)-(\omega \theta P)(P \pi)]\right) \\
& +\frac{\lambda_{1}}{2}\left(\pi^{2}-\frac{\alpha}{\omega^{2}}\right)+\lambda_{2}(\omega \pi) .
\end{aligned}
$$

The dynamics of basic variables is governed by Hamiltonian equations $\dot{z}=\{z, H\}$, where $z=(x, p, \omega, \pi)$, and the
Hamiltonian is given in (72). Equivalently, we can use the first-order variational problem equivalent to (56):

$$
\begin{aligned}
S_{H} & =\int d \tau p_{\mu} \dot{x}^{\mu}+\pi_{\mu} \dot{\omega}^{\mu} \\
& -\left[\frac{\lambda_{1}}{2}\left(P^{2}+(m c)^{2}+\pi^{2}-\frac{\alpha}{\omega^{2}}\right)+\lambda_{2}(\omega \pi)\right. \\
& \left.+\lambda_{3}(P \omega)+\lambda_{4}(P \pi)\right] .
\end{aligned}
$$

Variation with respect to $\lambda_{i}$ gives constraints (61) and (62), while variation with respect to $x, p, \omega$, and $\pi$ gives the dynamical equations. By construction of $S_{H}$, the variational equation $\delta S_{\kappa} / \delta p_{\mu}=0$ is equivalent to $\dot{x}^{\mu}=\left\{x^{\mu}, H\right\}$ and so on. The equations can be written in a manifestly covariant form as follows:

$$
\begin{aligned}
\dot{x}^{\mu} & =\lambda_{1}\left[P^{\mu}+2 a\left[(\pi \theta P) \omega^{\mu}-(\omega \theta P) \pi^{\mu}\right]\right], \\
\nabla P_{\mu} & =R_{\beta \mu \nu}^{\alpha} \pi_{\alpha} \omega^{\beta} \dot{x}^{\nu}, \\
\nabla \omega^{\mu} & =-2 \lambda_{1} a(\omega \theta P) P^{\mu}+\lambda_{2} \omega^{\mu}+\lambda_{1} \pi^{\mu}, \\
\nabla \pi_{\mu} & =-2 \lambda_{1} a(\pi \theta P) P_{\mu}-\lambda_{2} \pi_{\mu}-\lambda_{1} \frac{\omega_{\mu}}{\omega^{2}} .
\end{aligned}
$$

According to general theory $[29,36,37]$, neither constraints nor equations of motion determine the functions $\lambda_{1}$ and $\lambda_{2}$. Their presence in the equations of motion implies that evolution of our basic variables is ambiguous. This is in correspondence with two local symmetries presented in the model. The variables with ambiguous dynamics do not represent observable quantities, so we need to search for variables that can be candidates for observables. Consider antisymmetric tensor (50). As a consequence of $T_{3}=0$ and $T_{4}=0$, this obeys the Pirani supplementary condition $[4,5,7]$

$$
S^{\mu \nu} P_{v}=0 .
$$

Besides, the constraints $T_{2}$ and $T_{5}$ fix the value of square

$$
S^{\mu \nu} S_{\mu \nu}=8 \alpha,
$$

so we identify $S^{\mu v}$ with the Frenkel spin-tensor [9]. Equations (77) and (78) imply that only two components of spin-tensor are independent, as it should be for spin one-half particle. Equations of motion for $S^{\mu \nu}$ follow from (76). Besides, we 
express (74) and (75) in terms of the spin-tensor. This gives the system

$$
\begin{aligned}
\dot{x}^{\mu} & =\lambda_{1}\left[P^{\mu}+a S^{\mu \beta} \theta_{\beta \alpha} P^{\alpha}\right], \\
\nabla P_{\mu} & =-\frac{1}{4} R_{\mu \nu \alpha \beta} S^{\alpha \beta} \dot{x}^{\nu} \equiv-\frac{1}{4} \theta_{\mu \nu} \dot{x}^{\nu}, \\
\nabla S^{\mu \nu} & =2\left(P^{\mu} \dot{x}^{\nu}-P^{\nu} \dot{x}^{\mu}\right),
\end{aligned}
$$

where $\theta$ has been defined in (68). Equation (81), contrary to (76) for $\omega$ and $\pi$, does not depend on $\lambda_{2}$. This proves that the spin-tensor is invariant under local spin-plane symmetry. The remaining ambiguity due to $\lambda_{1}$ is related with reparametrization invariance and disappears when we work with physical dynamical variables $x^{i}(t)$. Equations (79)-(81), together with (77) and (78), form a closed system which determines evolution of a spinning particle.

To obtain the Hamiltonian equations, we can equally use the Dirac bracket constructed with help of second-class constraints:

$$
\begin{aligned}
\{A, B\}_{\mathrm{D}}= & \{A, B\} \\
& -\frac{1}{8 a}\left[\left\{A, T_{3}\right\}\left\{T_{4}, B\right\}-\left\{A, T_{4}\right\}\left\{T_{3}, B\right\}\right] .
\end{aligned}
$$

Since the Dirac bracket of a second-class constraint with any quantity vanishes, we can now omit $T_{3}$ and $T_{4}$ from (72); this yields the Hamiltonian

$$
H_{1}=\frac{\lambda_{1}}{2}\left(P^{2}+m^{2} c^{2}\right)+\frac{\lambda_{1}}{2}\left(\pi^{2}-\frac{\alpha}{\omega^{2}}\right)+\lambda_{2}(\omega \pi) .
$$

Then, (74)-(76) can be obtained according to the rule $\dot{z}=$ $\left\{z, H_{1}\right\}_{\mathrm{D}}$. The quantities $x^{\mu}, P^{\mu}$, and $S^{\mu \nu}$, being invariant under spin-plane symmetry, have vanishing brackets with the corresponding first-class constraints $T_{2}$ and $T_{5}$. So, obtaining equations for these quantities, we can omit the last two terms in $H_{1}$, arriving at the familiar relativistic Hamiltonian

$$
H_{2}=\frac{\lambda_{1}}{2}\left(P^{2}+m^{2} c^{2}\right)
$$

Equations (79)-(81) can be obtained according to the rule $\dot{z}=\left\{z, H_{2}\right\}_{\mathrm{D}}$. From (84), we conclude that our model describes spinning particle without gravimagnetic moment. The Hamiltonian with gravimagnetic moment $\kappa$ has been proposed by Khriplovich [25] adding nonminimal interaction $\left(\lambda_{1} / 2\right)(\kappa / 16) R_{\mu \nu \alpha \beta} S^{\mu \nu} S^{\alpha \beta}$ to the expression for $H_{2}$. The corresponding Lagrangian formulation will be constructed in Section 5.1.

Let us exclude momenta $P^{\mu}$ and the auxiliary variable $\lambda_{1}$ from the Hamiltonian equations. This yields second-order equation for the particle's position $x^{\mu}(\tau)$. To achieve this, we observe that (79) is linear on $P$.

$$
\dot{x}^{\mu}=\lambda_{1} T^{\mu}{ }_{\nu} P^{\nu}, \quad \text { with } T_{\nu}^{\mu}=\delta_{v}^{\mu}+a S^{\mu \alpha} \theta_{\alpha \nu} .
$$

Using the identity

$$
(S \theta S)^{\mu \nu}=-\frac{1}{2}(S \theta) S^{\mu \nu}, \quad \text { where } S \theta=S^{\alpha \beta} \theta_{\alpha \beta},
$$

we find inverse of the matrix $T^{\mu}{ }_{\nu}$ :

$$
\begin{aligned}
\widetilde{\mathscr{T}}^{\mu}{ }_{\nu} & =\delta^{\mu}{ }_{\nu}-\frac{1}{8 m^{2} c^{2}} S^{\mu \sigma} \theta_{\sigma v}, \\
T^{\mu}{ }_{\alpha} \widetilde{\mathscr{T}}^{\alpha}{ }_{\nu} & =\delta_{\nu}^{\mu},
\end{aligned}
$$

so $(85)$ can be solved with respect to $P^{\mu}, P^{\mu}=\left(1 / \lambda_{1}\right) \widetilde{\mathscr{T}}^{\mu}{ }_{v} \dot{x}^{\nu}$. We substitute $P^{\mu}$ into the constraint $P^{2}+m^{2} c^{2}=0$; this gives expression for $\lambda_{1}$ :

$$
\lambda_{1}=\frac{\sqrt{-G_{\mu \nu} \dot{x}^{\mu} \dot{x}^{\nu}}}{m c} \equiv \frac{\sqrt{-\dot{x} G \dot{x}}}{m c} .
$$

We have introduced the effective metric

$$
G_{\mu \nu} \equiv \widetilde{\mathscr{T}}_{\mu}^{\alpha} g_{\alpha \beta} \widetilde{\mathscr{T}}^{\beta}{ }_{\nu}
$$

The matrix $G$ is composed of the original metric $\eta_{\mu \nu}$ plus (spin- and field-dependent) contribution; $G_{\mu \nu}=\eta_{\mu \nu}+h_{\mu \nu}(S)$. So, we call $G$ the effective metric produced along the world line by interaction of spin with gravity. The effective metric will play the central role in our discussion of ultrarelativistic limit.

From (85) and (88), we obtain the final expression for $P_{\mu}$,

$$
\begin{aligned}
P^{\mu} & =\frac{m c}{\sqrt{-\dot{x} G \dot{x}}} \widetilde{\mathscr{T}}^{\mu}{ }_{\nu} \dot{x}^{\nu} \\
& =\frac{m c}{\sqrt{-\dot{x} G \dot{x}}}\left[\dot{x}^{\mu}-\frac{1}{8 m^{2} c^{2}} S^{\mu \nu} \theta_{\nu \sigma} \dot{x}^{\sigma}\right],
\end{aligned}
$$

and Lagrangian form of the Pirani condition,

$$
S_{\nu}^{\mu} \dot{x}^{\nu}-\frac{1}{8(m c)^{2}}(S S \theta \dot{x})^{\mu}=0 .
$$

Using (90) and (91) in (80) and (81), we finally obtain

$$
\begin{aligned}
\nabla\left[\frac{\widetilde{T}^{\mu}{ }_{\nu} \dot{x}^{\nu}}{\sqrt{-\dot{x} G \dot{x}}}\right] & =-\frac{1}{4 m c} R_{\nu \alpha \beta}^{\mu} S^{\alpha \beta} \dot{x}^{\nu}, \\
\nabla S^{\mu \nu} & =\frac{1}{4 m c \sqrt{-\dot{x} G \dot{x}}} \dot{x}^{[\mu} S^{\nu] \sigma} \theta_{\sigma \alpha} \dot{x}^{\alpha} .
\end{aligned}
$$

These equations, together with conditions (91) and (78), form closed system for the set $\left(x^{\mu}, S^{\mu \nu}\right)$. The consistency of constraints (91) and (78) with the dynamical equations is guaranteed by Dirac procedure for singular systems.

4.2. Lagrangian Action of Spinning Particle with Unfixed Value of Spin. Lagrangians (48) and (56) yield the fixed value of spin (78); that is, they correspond to an elementary particle. Let us present the modification which leads to the theory with unfixed spin and similarly to Hanson-Regge approach [22], with a mass-spin trajectory constraint. Consider the following Lagrangian in curved background: 


$$
L=-\frac{m c}{\sqrt{2}} \sqrt{-\dot{x} N \dot{x}-l^{2} \frac{\nabla \omega N \nabla \omega}{\omega^{2}}+\sqrt{\left[\dot{x} N \dot{x}+l^{2} \frac{\nabla \omega N \dot{\omega}}{\omega^{2}}\right]^{2}-4 l^{2} \frac{(\dot{x} N \nabla \omega)^{2}}{\omega^{2}}}},
$$

where $l$ is a parameter with the dimension of length. Applying the Dirac procedure as in Section 4.1, we obtain the Hamiltonian

$$
\begin{aligned}
H= & \frac{\lambda_{1}}{2}\left(P^{2}+m^{2} c^{2}+\frac{\pi^{2} \omega^{2}}{l^{2}}\right)+\lambda_{2}(\omega \pi)+\lambda_{3}(P \omega) \\
& +\lambda_{4}(P \pi),
\end{aligned}
$$

which turns out to be combination of the first-class constraints $P^{2}+m^{2} c^{2}+\pi^{2} \omega^{2} / l^{2}=0$ and $\omega \pi=0$ and the second-class constraints $P \omega=0$ and $P \pi=0$. The Dirac procedure stops on the first stage; that is, there are no secondary constraints. As compared with (56), the first-class constraint $\pi^{2}-\alpha / \omega^{2}=0$ does not appear in the present model. Due to this, square of spin is not fixed; $S^{2}=8\left(\omega^{2} \pi^{2}-\omega \pi\right) \approx$ $8 \omega^{2} \pi^{2}$. Using this equality, the mass-shell constraint acquires the string-like form

$$
P^{2}+m^{2} c^{2}+\frac{1}{8 l^{2}} S^{2}=0 .
$$

The model has four physical degrees of freedom in the spin-sector. As the independent gauge-invariant degrees of freedom, we can take three components $S^{i j}$ of the spin-tensor together with any one product of conjugate coordinates, for instance, $\omega^{0} \pi^{0}$.

Using the auxiliary variable $\lambda$, we can rewrite the Lagrangian in the equivalent form

$$
\begin{aligned}
L & =\frac{1}{2 \lambda}\left[\dot{x} N \dot{x}+l^{2} \frac{\nabla \omega N \nabla \omega}{\omega^{2}}\right. \\
& \left.-\sqrt{\left[\dot{x} N \dot{x}+l^{2} \frac{\nabla \omega N \dot{\omega}}{\omega^{2}}\right]^{2}-4 l^{2} \frac{(\dot{x} N \nabla \omega)^{2}}{\omega^{2}}}\right]-\frac{\lambda}{4} \\
& \cdot m^{2} c^{2} .
\end{aligned}
$$

Contrary to (94), it admits the massless limit.

4.3. Mathisson-Papapetrou-Tulczyjew-Dixon (MPTD) Equations and Dynamics of Representative Point of a Rotating Body. In this section, we discuss MPTD equations of a rotating body in the form studied by Dixon (our $S$ is twice of that of Dixon) (for the relation of the Dixon equations with those of Papapetrou and Tulczyjew, see page 335 in [5]),

$$
\begin{aligned}
\nabla P^{\mu} & =-\frac{1}{4} R^{\mu}{ }_{\nu \alpha \beta} S^{\alpha \beta} \dot{x}^{\nu} \equiv-\frac{1}{4}(\theta \dot{x})^{\mu}, \\
\nabla S^{\mu \nu} & =2\left(P^{\mu} \dot{x}^{\nu}-P^{\nu} \dot{x}^{\mu}\right), \\
S^{\mu \nu} P_{\nu} & =0,
\end{aligned}
$$

and compare them with equations of motion of our spinning particle. In particular, we show that the effective metric $G_{\mu \nu}$ also emerges in this formalism. MPTD equations appeared in multipole approach to description of a body [1-6], where the energy-momentum of the body is modelled by a set of multipoles. In this approach, $x^{\mu}(\tau)$ is called representative point of the body; we take it in arbitrary parametrization $\tau$ (contrary to Dixon, we do not assume the proper-time parametrization; i.e., we do not add the equation $g_{\mu \nu} \dot{x}^{\mu} \dot{x}^{\nu}=$ $-c^{2}$ to the system above). $S^{\mu \nu}(\tau)$ is associated with inner angular momentum, and $P^{\mu}(\tau)$ is called momentum. Firstorder equations (98) and (99) appear in the pole-dipole approximation, while algebraic equation (100) has been added by hand (for geometric interpretation of the spin supplementary condition in the multipole approach, see [5]). After that, the number of equations coincides with the number of variables.

To compare MPTD equations with those of Section 4.1, we first observe some useful consequences of system (98)(100).

Take derivative of the constraint, $\nabla\left(S^{\mu \nu} P_{\nu}\right)=0$, and use (98) and (99); this gives the expression

$$
(P \dot{x}) P^{\mu}=P^{2} \dot{x}^{\mu}+\frac{1}{8}(S \theta \dot{x})^{\mu},
$$

which can be written in the form

$$
P^{\mu}=\frac{P^{2}}{(P \dot{x})}\left(\delta^{\mu}{ }_{\nu}+\frac{1}{8 P^{2}}(S \theta)^{\mu}{ }_{\nu}\right) \dot{x}^{\nu} \equiv \frac{P^{2}}{(P \dot{x})} \widetilde{T}^{\mu}{ }_{\nu} \dot{x}^{\nu} .
$$

Contract (101) with $\dot{x}_{\mu}$. Taking into account that $(P \dot{x})<0$, this gives $(P \dot{x})=-\sqrt{-P^{2}} \sqrt{-\dot{x} \widetilde{T} \dot{x}}$. Using this in (102), we obtain

$$
\begin{aligned}
P^{\mu} & =\frac{\sqrt{-P^{2}}}{\sqrt{-\dot{x} \widetilde{T} \dot{x}}}(\widetilde{\mathscr{T}} \dot{x})^{\mu}, \\
\widetilde{\mathscr{T}}^{\mu}{ }_{\nu} & =\delta^{\mu}{ }_{\nu}+\frac{1}{8 P^{2}}(S \theta)^{\mu}{ }_{\nu} .
\end{aligned}
$$

For the latter use, we observe that in our model with composite $S^{\mu \nu}$ we used identity (86) to invert $T^{\mu}$; then Hamiltonian equation (79) has been written in the form of (90); the latter can be compared with (103).

Contracting (99) with $S_{\mu \nu}$ and using (100), we obtain $(d / d \tau)\left(S^{\mu \nu} S_{\mu \nu}\right)=0$; that is, square of spin is a constant of motion. Contraction of (101) with $P_{\mu}$ gives $(P S \theta \dot{x})=0$. Contraction of (101) with $(\dot{x} \theta)_{\mu}$ gives $(P \theta \dot{x})=0$. Contraction of (98) with $P_{\mu}$ gives $(d / d \tau)\left(P^{2}\right)=-(1 / 2)(P \theta \dot{x})=0$; that is, $P^{2}$ is one more constant of motion, say $k, \sqrt{-P^{2}}=k=$ const (in our model this is fixed as $k=m c$ ). Substituting (103) 
into (98)-(100), we now can exclude $P^{\mu}$ from these equations, modulo to the constant of motion $k=\sqrt{-P^{2}}$.

Thus, square of momentum can not be excluded from system (98)-(101); that is, MPTD equations in this form do not represent a Hamiltonian system for the pair $x^{\mu}, P^{\mu}$. To improve this point, we note that (103) acquires a conventional form (as the expression for conjugate momenta of $x^{\mu}$ in the Hamiltonian formalism) if we add to system (98)-(100) one more equation, which fixes the remaining quantity $P^{2}$ (Dixon noticed this for the body in electromagnetic field; see his equation (4.5) in [6]). To see how the equation could look, we note that for nonrotating body (pole approximation) we expect equations of motion of spinless particle; $\nabla p^{\mu}=0$, $p^{\mu}=(m c / \sqrt{-\dot{x} g \dot{x}}) \dot{x}^{\mu}$, and $p^{2}+(m c)^{2}=0$. Independent equations of system (98)-(101) in this limit read $\nabla P^{\mu}=0$, $P^{\mu}=\left(\sqrt{-P^{2}} / \sqrt{-\dot{x} g \dot{x}}\right) \dot{x}^{\mu}$. Comparing the two systems, we see that the missing equation is the mass-shell condition $P^{2}+(m c)^{2}=0$. Returning to the pole-dipole approximation, an admissible equation should be $P^{2}+(m c)^{2}+f(S, \ldots)=0$, where $f$ must be a constant of motion. Since the only constant of motion in arbitrary background is $S^{2}$, we have finally

$$
P^{2}=-(m c)^{2}-f\left(S^{2}\right) .
$$

With this value of $P^{2}$, we can exclude $P^{\mu}$ from MPTD equations, obtaining closed system with second-order equation for $x^{\mu}$ (so, we refer to the resulting equations as Lagrangian form of MPTD equations). We substitute (103) into (98)(100); this gives

$$
\begin{aligned}
\nabla \frac{(\widetilde{\mathscr{T}} \dot{x})^{\mu}}{\sqrt{-\dot{x} \widetilde{T} \dot{x}}} & =-\frac{1}{4 \sqrt{-P^{2}}}(\theta \dot{x})^{\mu}, \\
\nabla S^{\mu \nu} & =-\frac{1}{4 \sqrt{-P^{2}} \sqrt{-\dot{x} \widetilde{T} \dot{x}}} \dot{x}^{[\mu}(S \theta \dot{x})^{\nu]}, \\
(S S \theta \dot{x})^{\mu} & =-8 P^{2}(S \dot{x})^{\mu}
\end{aligned}
$$

where (104) is implied. They determine evolution of $x^{\mu}$ and $S^{\mu \nu}$ for each given function $f\left(S^{2}\right)$.

It is convenient to introduce the effective metric $\mathscr{G}$ composed of the "tetrad field" $\widetilde{\mathscr{T}}^{\mu}$ :

$$
\mathscr{G}_{\mu \nu} \equiv g_{\alpha \beta} \widetilde{\mathscr{T}}_{\mu}^{\alpha} \widetilde{\mathscr{T}}_{\nu}^{\beta} .
$$

Equation (107) implies the identity

$$
\dot{x} \widetilde{\mathscr{T}} \dot{x}=\dot{x} \mathscr{G} \dot{x}
$$

so we can replace $\sqrt{-\dot{x} \widetilde{T} \dot{x}}$ in (105)-(107) by $\sqrt{-\dot{x} \mathscr{G} \dot{x}}$.
In resume, we have presented MPTD equations in the form

$$
\begin{gathered}
P^{\mu}=\frac{\sqrt{-P^{2}}}{\sqrt{-\dot{x} \mathscr{G} \dot{x}}}(\widetilde{T} \dot{x})^{\mu}, \\
\nabla P^{\mu}=-\frac{1}{4}(\theta \dot{x})^{\mu}, \\
\nabla S^{\mu \nu}=2 P^{[\mu} \dot{x}^{\nu]}, \\
S^{\mu \nu} P_{\nu}=0, \\
P^{2}+(m c)^{2}+f\left(S^{2}\right)=0, \\
S^{2} \text { is a constant of motion, }
\end{gathered}
$$

with $\widetilde{\mathscr{T}}^{\mu}{ }_{\nu}$ given in (103). Now, we are ready to compare them with Hamiltonian equations of our spinning particle, which we write here in the form

$$
\begin{aligned}
P^{\mu} & =\frac{m c}{\sqrt{-\dot{x} G \dot{x}}}(\widetilde{T} \dot{x})^{\mu}, \\
\nabla P^{\mu} & =-\frac{1}{4}(\theta \dot{x})^{\mu}, \\
\nabla S^{\mu \nu} & =2 P^{[\mu} \dot{x}^{\nu]}, \\
S^{\mu \nu} P_{\nu} & =0, \\
P^{2}+(m c)^{2} & =0, \\
S^{2} & =8 \alpha,
\end{aligned}
$$

with $\widetilde{\mathscr{T}}^{\mu}{ }_{\nu}$ given in (87). Comparing the systems, we see that our spinning particle has fixed values of spin and canonical momentum, while for MPTD particle the spin is a constant of motion and momentum is a function of spin. We conclude that all the trajectories of a body with given $m$ and $S^{2}=\beta$ are described by our spinning particle with spin $\alpha=\beta / 8$ and with the mass equal to $\sqrt{m^{2}-f^{2}(\beta) / c^{2}}$. In this sense, our spinning particle is equivalent to MPTD particle.

We point out that our final conclusion remains true even if we do not add (104) to MPTD equations; to study the class of trajectories of a body with $\sqrt{-P^{2}}=k$ and $S^{2}=\beta$, we take our spinning particle with $m=k / c$ and $\alpha=\beta / 8$.

MPTD equations in the Lagrangian form in (105)-(107) can be compared with (91)-(93).

4.4. Ultrarelativistic Limit: The Problems with MPTD Equations. The equations for trajectory (92) and for precession of spin (93) became singular at critical velocity which obeys the equation

$$
\dot{x} G \dot{x}=0 .
$$

As we discussed in Introduction, the singularity determines behavior of the particle in ultrarelativistic limit. In (114), effective metric (89) appeared instead of the original metric $g_{\mu \nu}$. It should be noted that the incorporation of constraints 
(62) and (66) into a variational problem, as well as the search for an interaction consistent with them, represents very strong restrictions on possible form of the Lagrangian. So, the appearance of effective metric seems to be unavoidable in a systematically constructed model of spinning particle. The same conclusion follows from our analysis of MPTD equations in Section 4.3.

The effective metric is composed of the original one plus (spin- and field-dependent) contribution; $G=g+h(S)$. So, we need to decide which of them the particle probes as the spacetime metric. Let us consider separately the two possibilities.

Let us use $g$ to define the three-dimensional geometry in (5)-(8). This leads to two problems. The first problem is that the critical speed turns out to be slightly more than the speed of light. To see this, we use the Pirani condition to write (114) in the form

$$
\begin{aligned}
-\left(\frac{d t}{d x^{0}}\right)^{2} \dot{x} G \dot{x} & =\left(c^{2}-\mathbf{v} \gamma \mathbf{v}\right)+\frac{1}{\left(2 m^{2} c^{2}\right)^{2}}(v \theta S S \theta v) \\
& =0
\end{aligned}
$$

with $v^{\mu}$ defined in (10). Using the expression $S^{\mu \nu}=2 \omega^{[\mu} \pi^{\nu]}$, we obtain

$$
\begin{aligned}
& -\left(\frac{d t}{d x^{0}}\right)^{2} \dot{x} G \dot{x} \\
& =\left(c^{2}-\mathbf{v} \gamma \mathbf{v}\right)+\frac{1}{\left(m^{2} c^{2}\right)^{2}}\left(\pi^{2}(v \theta \omega)^{2}+\omega^{2}(v \theta \pi)^{2}\right) \\
& =0 .
\end{aligned}
$$

As $\pi$ and $\omega$ are space-like vectors (see the discussion below (70)), the last term is nonnegative; this implies $\left|\mathbf{v}_{\mathrm{cr}}\right| \geq c$. Let us confirm that generally this term is nonvanishing function of velocity; then $\left|\mathbf{v}_{\mathrm{cr}}\right|>c$. Assume the contrary that this term vanishes at some velocity; then

$$
\begin{aligned}
& v \theta \omega=\theta_{0 i} \omega^{i}+\theta_{i 0} v^{i} \omega^{0}=0, \\
& v \theta \pi=\theta_{0 i} \pi^{i}+\theta_{i 0} v^{i} \pi^{0}=0 .
\end{aligned}
$$

We analyze these equations in the following special case. Consider a space with covariantly constant curvature $\nabla_{\mu} R_{\mu \nu \alpha \beta}=$ 0 . Then, $(d / d \tau)\left(\theta_{\mu \nu} S^{\mu \nu}\right)=2 \theta_{\mu \nu} \nabla S^{\mu \nu}$, and using (93) we conclude that $\theta_{\mu \nu} S^{\mu \nu}$ is an integral of motion. We further assume that the only nonvanishing part is the electric [38] part of the curvature, $R_{0 i 0 j}=K_{i j}$, with $\operatorname{det} K_{i j} \neq 0$. Then, the integral of motion acquires the form

$$
\theta_{\mu \nu} S^{\mu \nu}=2 K_{i j} S^{0 i} S^{0 j}
$$

Let us take the initial conditions for spin such that $K_{i j} S^{0 i} S^{0 j} \neq$ 0 ; then this holds at any future instant. Contrary to this, system (117) implies $K_{i j} S^{0 i} S^{0 j}=0$. Thus, the critical speed does not always coincide with the speed of light and, in general case, we expect that $\mathbf{v}_{\mathrm{cr}}$ is both field- and spindependent quantity.
The second problem is that acceleration of MPTD particle grows up in the ultrarelativistic limit. In the spinless limit (92) turn into the geodesic equation. Spin causes deviations from the geodesic equation due to right hand side of this equation, as well as due to the presence of the tetrad field $\widetilde{\mathscr{T}}^{\mu}{ }_{\nu}$ and of the effective metric $G$ in the left hand side. Due to the dependence of the tetrad field on the spin-tensor $S$, the singularity presented in (93) causes the appearance of the term proportional to $1 / \sqrt{\dot{x} G \dot{x}}$ in the expression for longitudinal acceleration. In the result, the acceleration grows up to infinity as the particle's speed approximates to the critical speed. To see this, we separate derivative of $\widetilde{\mathscr{T}}^{\mu}{ }_{\nu}$ in (92).

$$
\begin{aligned}
\nabla\left[\frac{\dot{x}^{\mu}}{\sqrt{-\dot{x} G \dot{x}}}\right]= & -T^{\mu}{ }_{\alpha}\left(\nabla \widetilde{\mathscr{T}}_{\beta}^{\alpha}\right) \frac{\dot{x}^{\beta}}{\sqrt{-\dot{x} G \dot{x}}} \\
& -\frac{1}{4 m c} T^{\mu}{ }_{\nu}(\theta \dot{x})^{\nu} .
\end{aligned}
$$

Using (93), we obtain

$$
\begin{aligned}
& {\left[\nabla \widetilde{\mathscr{T}}^{\mu}{ }_{\nu}\right] \dot{x}^{\nu}} \\
& \quad=-\frac{S^{\mu \alpha}}{8 m^{2} c^{2}}\left[\frac{R_{\alpha \nu \beta \sigma} \dot{x}^{\beta}(S \theta \dot{x})^{\sigma}}{2 m c \sqrt{-\dot{x} G \dot{x}}}+S^{\beta \sigma}\left(\nabla R_{\alpha \nu \beta \sigma}\right)\right] \dot{x}^{\nu} .
\end{aligned}
$$

Using this expression together with the identity $(T S)^{\mu \nu}=$ $8 m^{2} c^{2} a S^{\mu \nu},(120)$ reads

$$
\frac{d}{d \tau}\left[\frac{\dot{x}^{\mu}}{\sqrt{-\dot{x} G \dot{x}}}\right]=\frac{f^{\mu}}{\sqrt{-\dot{x} G \dot{x}}},
$$

where we denoted

$$
\begin{aligned}
f^{\mu} \equiv & a S^{\mu \alpha}\left[\frac{R_{\alpha \nu \beta \sigma} \dot{x}^{\beta}(S \theta \dot{x})^{\sigma}}{2 m c \sqrt{-\dot{x} G \dot{x}}}+S^{\beta \sigma}\left(\nabla R_{\alpha \nu \beta \sigma}\right)\right] \dot{x}^{\nu} \\
& -(\Gamma \dot{x} \dot{x})^{\mu}-\frac{\sqrt{-\dot{x} G \dot{x}}}{4 m c}(T \theta \dot{x})^{\mu} .
\end{aligned}
$$

It will be sufficient to consider static metric $g_{\mu \nu}(\mathbf{x})$ with $g_{0 i}=$ 0 . Then three-dimensional metric and velocity are

$$
\begin{aligned}
\gamma_{i j} & =g_{i j}, \\
v^{i} & =\frac{c}{\sqrt{-g_{00}}} \frac{d x^{i}}{d x^{0}} .
\end{aligned}
$$

Taking $\tau=x^{0}$, the spacial part of (122) with this metric reads

$$
\left(\frac{d t}{d x^{0}}\right)^{-1} \frac{d}{d x^{0}}\left[\frac{v^{i}}{\sqrt{-v G v}}\right]=\frac{f^{i}(v)}{\sqrt{-v G v}},
$$

with $v^{\mu}$ defined in (10), for the case

$$
\begin{aligned}
v^{\mu} & =\left(\frac{c}{\sqrt{-g_{00}}}, \mathbf{v}\right), \\
-v G v & =-v \widetilde{\mathscr{T}} v=c^{2}-\mathbf{v} g \mathbf{v}+\frac{(v S \theta v)}{8 m^{2} c^{2}} .
\end{aligned}
$$


In the result, we have presented the equation for trajectory in the form convenient for analysis of acceleration; see (29). Using the definition of three-dimensional covariant derivative (25), we present the derivative on the l.h.s. of (125) as follows:

$$
\begin{gathered}
\frac{d}{d x^{0}}\left[\frac{v^{i}}{\sqrt{-v G v}}\right]=\frac{1}{\sqrt{-v G v}}\left[\mathscr{M}_{k}^{i} \nabla_{0} v^{k}\right. \\
\left.-\widetilde{\Gamma}(\gamma)_{j k}^{i} v^{j} v^{k} \frac{d t}{d x^{0}}+\frac{K v^{i}}{2(-v G v)}\right] .
\end{gathered}
$$

We have denoted

$$
\begin{aligned}
K & =\left(\nabla_{0} G_{\mu \nu}\right) v^{\mu} v^{\nu}-v^{\mu} G_{\mu 0} v^{k} \partial_{k} \ln \left(-g_{00}\right), \\
\mathscr{M}_{k}^{i} & =\delta_{k}^{i}-\frac{v^{i} v^{\mu} G_{\mu k}}{v G v} .
\end{aligned}
$$

The matrix $\mathscr{M}_{k}^{i}$ has the inverse

$$
\begin{aligned}
\widetilde{\mathscr{M}}_{k}^{i} & =\delta^{i}{ }_{k}+\frac{v^{i} v^{\mu} G_{\mu k}}{v^{\sigma} G_{\sigma 0} v^{0}}, \\
\text { then } \widetilde{\mathscr{M}}_{k}^{i} v^{k} & =v^{i} \frac{v G v}{v^{\sigma} G_{\sigma 0} v^{0}} .
\end{aligned}
$$

Combining these equations, we obtain the three-acceleration of our spinning particle:

$$
\begin{aligned}
a^{i} & =\left(\frac{d t}{d x^{0}}\right)^{-1} \nabla_{0} v^{i} \\
& =\widetilde{\mathscr{M}}_{k}^{i}\left[f^{k}+(\widetilde{\Gamma} v)^{k}\right]+\frac{K v^{i}}{2 v^{\sigma} G_{\sigma 0}} .
\end{aligned}
$$

Finally, using manifest form of $f^{i}$ from (123), we have

$$
\begin{aligned}
a^{i}= & \frac{\widetilde{M}_{k}^{i} \widehat{S}^{k}}{\sqrt{-v G v}}-c^{2} \widetilde{\mathscr{M}}_{k}^{i} \frac{\gamma^{k j} \partial_{j} g_{00}}{2 g_{00}} \\
& -\frac{\sqrt{-v G v}}{4 m c} \widetilde{\mathscr{M}}_{k}^{i}(T \theta v)^{k}+\frac{K v^{i}}{2 v^{\sigma} G_{\sigma 0}} \\
& +\widetilde{a \mathscr{M}}_{k}^{i} S^{k \alpha} R_{\alpha \nu \beta \sigma ; \lambda} S^{\beta \sigma} v^{v} v^{\lambda} .
\end{aligned}
$$

The longitudinal acceleration is obtained by projecting $a^{i}$ on the direction of velocity, that is,

$$
\begin{aligned}
(\mathbf{v} \gamma \mathbf{a})= & \frac{a c(\mathbf{v} \gamma \widetilde{\mathscr{M}})_{k} \widehat{S}^{k}}{2 m \sqrt{-v G v}}-c^{2}(\mathbf{v} \gamma \widetilde{\mathscr{M}})_{k} \frac{\gamma^{k j} \partial_{j} g_{00}}{2 g_{00}} \\
& -\frac{\sqrt{-v G v}}{4 m c}(\mathbf{v} \gamma \widetilde{\mathscr{M}})_{k}(T \theta v)^{k}+\frac{K}{2 v^{\sigma} G_{\sigma 0}}(\mathbf{v} \gamma \mathbf{v}) \\
& +a(\mathbf{v} \gamma \widetilde{\mathscr{M}})_{k} S^{k \alpha} R_{\alpha \nu \beta \sigma ; \lambda} S^{\beta \sigma} v^{v} v^{\lambda}
\end{aligned}
$$

where $\widehat{S}^{k}=S^{k \mu} R_{\mu \nu \alpha \beta} v^{\nu} v^{\alpha}(S \theta v)^{\beta}$. As the speed of the particle gets closer to the critical velocity, the longitudinal acceleration diverges due to the first term in (132). In resume, assuming that MPTD particle sees the original geometry $g_{\mu \nu}$, we have a theory with unsatisfactory behavior in the ultrarelativistic limit.

Let us consider the second possibility; that is, we take $G_{\mu \nu}$ to construct three-dimensional geometry (5)-(8). With these definitions we have, by construction, $-\dot{x} G \dot{x}=\left(d t / d x^{0}\right)^{2}\left(c^{2}-\right.$ $(\mathbf{v} \gamma \mathbf{v}))$, so the critical speed coincides with the speed of light. In the present case, the expression for three-acceleration can be obtained in closed form for an arbitrary curved background. Taking $\tau=x^{0}$, the spacial part of (122) implies

$$
\left(\frac{d t}{d x^{0}}\right)^{-1} \frac{d}{d x^{0}}\left[\frac{v^{i}}{\sqrt{c^{2}-\mathbf{v} \gamma \mathbf{v}}}\right]=\frac{f^{i}(v)}{\sqrt{c^{2}-\mathbf{v} \gamma \mathbf{v}}}
$$

where, from (123), $f^{i}$ is given by

$$
\begin{aligned}
f^{i} \equiv & a S^{i \alpha}\left[\frac{R_{\alpha \nu \beta \sigma} v^{\beta}(S \theta v)^{\sigma}}{2 m c \sqrt{c^{2}-\mathbf{v} \gamma \mathbf{v}}}+S^{\beta \sigma}\left(\nabla R_{\alpha \nu \beta \sigma}\right)\right] v^{v} \\
& -\Gamma_{\mu \nu}^{i}(G) v^{\mu} v^{l} v-\frac{\sqrt{c^{2}-\mathbf{v} \gamma \mathbf{v}}}{4 m c}(T \theta v)^{i} .
\end{aligned}
$$

Equation (133) is of the form in (29), so the acceleration is given by (38) and (39), where, for the present case, $\gamma_{i j}=G_{i j}-$ $G_{0 i} G_{0 j} / G_{00}$.

$$
\begin{aligned}
a^{i} & =\widetilde{M}_{j}^{i}\left[f^{j}+\widetilde{\Gamma}_{k l}^{j}(\gamma) v^{k} v^{l}\right]+\frac{1}{2}\left(\frac{d t}{d x^{0}}\right)^{-1} \\
\cdot & {\left[\left(\mathbf{v} \partial_{0} \gamma \gamma^{-1}\right)^{i}-\frac{\left(\mathbf{v} \partial_{0} \gamma \mathbf{v}\right)}{c^{2}} v^{i}\right], } \\
\mathbf{v} \gamma \mathbf{a} & =\left(1-\frac{\mathbf{v} \gamma \mathbf{v}}{c^{2}}\right)\left[(\mathbf{v} \gamma)_{i}\left[f^{i}(v)+\widetilde{\Gamma}_{k l}^{i}(\gamma) v^{k} v^{l}\right]\right. \\
& \left.+\frac{1}{2}\left(\frac{d t}{d x^{0}}\right)^{-1}\left(\mathbf{v} \partial_{0} \gamma \mathbf{v}\right)\right] .
\end{aligned}
$$

With $f^{i}$ given in (134), the longitudinal acceleration vanishes as $v \rightarrow c$.

Let us resume the results of this subsection. Assuming that spinning particle probes the three-dimensional spacetime geometry determined by the original metric $g$, we have a theory with unsatisfactory ultrarelativistic limit. First, the critical speed, which the particle can not overcome during its evolution in gravitational field, can be more than the speed of light. Second, the longitudinal acceleration grows up to infinity in the ultrarelativistic limit. Assuming that the the particle sees the effective metric $G(S)$ as the space-time metric, we avoided the two problems. But the resulting theory still possesses the problem. Acceleration (135) contains the singularity due to $f^{i} \sim 1 / \sqrt{c^{2}-(\mathbf{v} \gamma \mathbf{v})}$; that is, at $v=c$ the acceleration becomes orthogonal to the velocity but remains divergent. We conclude that MPTD equations do not seem promising candidate for description of a relativistic rotating body. 


\section{Nonminimal Interaction with Gravitational Field}

Can we modify the MPTD equations to obtain a theory with reasonable behavior with respect to the original metric $g_{\mu \nu}$ ? In the previous section we have noticed that the bad behavior of acceleration originates from the fact that variation rate of spin (93) diverges in the ultrarelativistic limit, $\nabla S \sim$ $1 / \sqrt{\dot{x} G \dot{x}}$, and contributes to expression for acceleration (132) through the tetrad field $\widetilde{T}_{\nu}^{\mu}(S)$. To improve this, we note that MPTD equations result from minimal interaction of spinning particle with gravitational field. In this section, we demonstrate that vector model of spin admits also a nonminimal interaction which involves the interaction constant $\kappa$. By analogy with the magnetic moment, the interaction constant $\kappa$ is called gravimagnetic moment [25]. In the resulting theory, the equation for precession of spin, $\nabla S \sim 1 / \sqrt{-\dot{x} G \dot{x}}$, is replaced by $\nabla S \sim \sqrt{-\dot{x} g \dot{x}}$. This improves the bad behavior of MPTD equations. As it will be seen below, introducing $\kappa$ we effectively change the supplementary spin condition and hence the definition of center of mass.

5.1. Hamiltonian Variational Problem. We add the term $\left(\lambda_{1} / 2\right) \kappa R_{\alpha \beta \mu \nu} \omega^{\alpha} \pi^{\beta} \omega^{\mu} \pi^{\nu} \equiv\left(\lambda_{1} / 2\right)(\kappa / 16) \theta S$ into Hamiltonian action (73). Thus, we consider the variational problem

$$
\begin{aligned}
S_{\kappa} & =\int d \tau p_{\mu} \dot{x}^{\mu}+\pi_{\mu} \dot{\omega}^{\mu}-\left[\frac { \lambda _ { 1 } } { 2 } \left(P^{2}\right.\right. \\
& \left.+\kappa R_{\alpha \beta \mu \nu} \omega^{\alpha} \pi^{\beta} \omega^{\mu} \pi^{\nu}+(m c)^{2}+\pi^{2}-\frac{\alpha}{\omega^{2}}\right) \\
& \left.+\lambda_{2}(\omega \pi)+\lambda_{3}(P \omega)+\lambda_{4}(P \pi)\right]
\end{aligned}
$$

on the space of independent variables $x^{\mu}, p_{\nu}, \omega^{\mu}, \pi_{\nu}$, and $\lambda_{a}$. We have denoted $P_{\mu} \equiv p_{\mu}-\Gamma_{\alpha \mu}^{\beta} \omega^{\alpha} \pi_{\beta}, P^{2}=g^{\mu \nu} P_{\mu} P_{\nu}$, and so on. Note also that the first two terms can be identically rewritten in the general-covariant form $p_{\mu} \dot{x}^{\mu}+\pi_{\mu} \dot{\omega}^{\mu}=P_{\mu} \dot{x}^{\mu}+\pi_{\mu} \nabla \omega^{\mu}$. Variation of the action with respect to $\lambda_{a}$ gives the algebraic equations

$$
\begin{aligned}
P^{2}+\kappa R_{\alpha \beta \mu \nu} \omega^{\alpha} \pi^{\beta} \omega^{\mu} \pi^{\nu}+(m c)^{2}+\pi^{2}-\frac{\alpha}{\omega^{2}} & =0, \\
\omega \pi & =0, \\
P \omega & =0, \\
P \pi & =0,
\end{aligned}
$$

while variations with respect to the remaining variables yield dynamical equations which can be written in the covariant form as follows (note that, by construction of $S_{\kappa}$, the variational equation $\delta S_{\kappa} / \delta p_{\mu}=0$ is equivalent to $\dot{x}^{\mu}=$ $\left\{x^{\mu}, H\right\}$ and so on):

$$
\begin{aligned}
\frac{\delta S_{\kappa}}{\delta p_{\mu}} & =0 \Longleftrightarrow \\
\dot{x}^{\mu} & =\lambda_{1} P^{\mu}+\lambda_{3} \omega^{\mu}+\lambda_{4} \pi^{\mu},
\end{aligned}
$$

$$
\begin{aligned}
& \frac{\delta S_{\kappa}}{\delta x^{\mu}}=0 \Longleftrightarrow \\
& \nabla P_{\mu}=-R_{\mu \nu \alpha \beta} \dot{x}^{\nu} \omega^{\alpha} \pi^{\beta}-\frac{1}{2} \lambda_{1} \kappa \nabla_{\mu} R_{\sigma \nu \alpha \beta} \omega^{\sigma} \pi^{\nu} \omega^{\alpha} \pi^{\beta}, \\
& \frac{\delta S_{\kappa}}{\delta \pi_{\mu}}=0 \Longleftrightarrow \\
& \nabla \omega^{\mu}=\lambda_{1} \pi^{\mu}-\lambda_{1} \kappa R^{\mu}{ }_{\alpha \beta \nu} \omega^{\alpha} \omega^{\beta} \pi^{\nu}+\lambda_{2} \omega^{\mu}+\lambda_{4} P^{\mu}, \\
& \frac{\delta S_{\kappa}}{\delta \omega^{\mu}}=0 \Longleftrightarrow \\
& \nabla \pi_{\mu}=-\frac{\lambda_{1} \alpha}{\omega^{4}} \omega_{\mu}-\lambda_{1} \kappa R_{\mu \nu \alpha \beta} \pi^{\nu} \omega^{\alpha} \pi^{\beta}-\lambda_{2} \pi_{\mu}-\lambda_{3} P_{\mu} .
\end{aligned}
$$

Equation (140) has been repeatedly used to obtain the final form in (141)-(143) of the equations $\delta S_{\kappa} / \delta x^{\mu}=0$, $\delta S_{\kappa} / \delta \pi_{\mu}=0$, and $\delta S_{\kappa} / \delta \omega^{\mu}=0$. Computing time derivative of algebraic equations (139) and using (140)-(143), we obtain the consequences

$$
\begin{aligned}
\pi^{2} & -\frac{\alpha}{\omega^{2}}=0, \\
\lambda_{3} & =4 a \lambda_{1}\left[2(1-\kappa) R_{\alpha \beta \mu \nu} \omega^{\alpha} \pi^{\beta} \pi^{\mu} P^{\nu}\right. \\
& \left.+\kappa \pi^{\sigma}\left(\nabla_{\sigma} R_{\mu \nu \alpha \beta}\right) \omega^{\mu} \pi^{\nu} \omega^{\alpha} \pi^{\beta}\right], \\
\lambda_{4} & =-4 a \lambda_{1}\left[2(1-\kappa) R_{\alpha \beta \mu \nu} \omega^{\alpha} \pi^{\beta} \omega^{\mu} P^{\nu}\right. \\
& \left.+\kappa \omega^{\sigma}\left(\nabla_{\sigma} R_{\mu \nu \alpha \beta}\right) \omega^{\mu} \pi^{\nu} \omega^{\alpha} \pi^{\beta}\right] .
\end{aligned}
$$

Here and below we use the following notation. In the equation which relates velocity and momentum will appear the matrix $\mathscr{T}_{\nu}^{\mu}$ :

$$
\begin{aligned}
\mathscr{T}^{\alpha}{ }_{\nu} & \equiv \delta^{\alpha}{ }_{\nu}-(\kappa-1) a S^{\alpha \sigma} \theta_{\sigma \nu}, \\
a & =\frac{2}{16 m^{2} c^{2}+(\kappa+1)(S \theta)} .
\end{aligned}
$$

The matrix has an inverse given by

$$
\begin{gathered}
{\widetilde{T}^{\alpha}}_{\nu} \equiv \delta^{\alpha}{ }_{\nu}+(\kappa-1) b S^{\alpha \sigma} \theta_{\sigma \nu}, \\
b=\frac{1}{8 m^{2} c^{2}+\kappa(S \theta)} .
\end{gathered}
$$

The vector $Z^{\mu}$ is defined by

$$
Z^{\mu}=\frac{b}{8 c} S^{\mu \sigma}\left(\nabla_{\sigma} R_{\alpha \beta \rho \delta}\right) S^{\alpha \beta} S^{\rho \delta} \equiv \frac{b}{8 c} S^{\mu \sigma} \nabla_{\sigma}(S \theta) .
$$

This vanishes in a space with homogeneous curvature, $\nabla R=$ 0 .

The time derivatives of (138), (144), and (145) do not yield new algebraic equations. Due to (144), we can replace constraint (138) on $P^{2}+\kappa R_{\alpha \beta \mu \nu} \omega^{\alpha} \pi^{\beta} \omega^{\mu} \pi^{\nu}+(m c)^{2}=0$. The obtained expressions for $\lambda_{3}$ and $\lambda_{4}$ can be used to exclude these variables from (140)-(143). 
The constraints and equations of motion do not determine the functions $\lambda_{1}$ and $\lambda_{2}$; that is, the nonminimal interaction preserves both reparametrization and spin-plane symmetries of the theory. The presence of $\lambda_{1}$ and $\lambda_{2}$ in (142) and (143) implies that evolution of the basic variables is ambiguous, so they are not observable. To find the candidates for observables, we note once again that (142) and (143) imply an equation for $S^{\mu \nu}$ which does not contain $\lambda_{2}$. So, we rewrite (140) and (141) in terms of spin-tensor and add to them the equation for $S^{\mu \nu}$; this gives the system

$$
\begin{aligned}
\dot{x}^{\mu} & =\lambda_{1}\left[\mathscr{T}^{\mu}{ }_{\nu} P^{\nu}+\kappa \frac{a c}{b} Z^{\mu}\right], \\
\nabla P_{\mu} & =-\frac{1}{4} \theta_{\mu \nu} \dot{x}^{\nu}-\frac{\lambda_{1} \kappa}{32} \nabla_{\mu}(S \theta), \\
\nabla S^{\mu \nu} & =-\frac{\kappa \lambda_{1}}{4}(\theta S)^{[\mu \nu]}+2 P^{[\mu} \dot{x}^{\nu]} .
\end{aligned}
$$

Besides, constraints (138), (139), and (144) imply

$$
\begin{aligned}
P^{2}+\frac{\kappa}{16} \theta S+(m c)^{2} & =0, \\
S^{\mu \nu} P_{\nu} & =0, \\
S^{\mu \nu} S_{\mu \nu} & =8 \alpha .
\end{aligned}
$$

Equations (153) imply that only two components of spintensor are independent, as it should be for spin one-half particle. Equation (151), contrary to the equations for $\omega$ and $\pi$, does not depend on $\lambda_{2}$. This proves that the spin-tensor is invariant under local spin-plane symmetry. The remaining ambiguity due to $\lambda_{1}$ is related to reparametrization invariance and disappears when we work with physical dynamical variables $x^{i}(t)$. Equations (149)-(151), together with (152) and (153), form a closed system which determines evolution of the spinning particle.

The gravimagnetic moment $\kappa$ can generally take any value. When $\kappa=0$, we recover the MPTD equations.

Let us exclude $P^{\mu}$ and $\lambda_{1}$ from (150) and (151). Using (147) we solve (149) with respect to $P^{\mu}$. Using the resulting expression in constraint (152), we obtain $\lambda_{1}$ :

$$
\begin{aligned}
\lambda_{1} & =\frac{\sqrt{-\dot{x} \mathscr{G} \dot{x}}}{m_{r} c}, \\
\text { with } m_{r}^{2} & \equiv m^{2}+\frac{\kappa}{16 c^{2}}(S \theta)-\kappa^{2} Z^{2},
\end{aligned}
$$

where the effective metric now is given by

$$
\mathscr{G}_{\mu \nu}=\widetilde{\mathscr{T}}_{\mu}^{\alpha} g_{\alpha \beta} \widetilde{\mathscr{T}}_{\nu}^{\beta} \text {. }
$$

Then the expression for momentum in terms of velocity implied by (149) is

$$
P^{\mu}=\frac{m_{r} c}{\sqrt{-\dot{x} \mathscr{G} \dot{x}}} \widetilde{\mathscr{T}}^{\mu} \dot{x}^{\nu}-\kappa c Z^{\mu} .
$$

We substitute this $P^{\mu}$ into (150) and (151):

$$
\begin{aligned}
\nabla\left[\frac{m_{r}}{\sqrt{-\dot{x} \mathscr{G} \dot{x}}} \widetilde{\mathscr{T}}^{\mu}{ }_{\nu} \dot{x}^{\nu}\right]= & -\frac{1}{4 c} \theta^{\mu}{ }_{\nu} \dot{x}^{\nu} \\
& -\kappa \frac{\sqrt{-\dot{x} \mathscr{G} \dot{x}}}{32 m_{r} c^{2}} \nabla^{\mu}(S \theta) \\
& +\kappa \nabla Z^{\mu}, \\
\nabla S^{\mu \nu}= & -\frac{\kappa \sqrt{-\dot{x} \mathscr{G} \dot{x}}}{4 m_{r} c}(\theta S)^{[\mu \nu]} \\
& -\frac{2 m_{r} c(\kappa-1) b}{\sqrt{-\dot{x} \mathscr{G} \dot{x}}} \dot{x}^{[\mu}(S \theta \dot{x})^{\nu]} \\
& +2 \kappa c \dot{x}^{[\mu} Z^{\nu]} .
\end{aligned}
$$

Together with (153), this gives us the Lagrangian equations for the spinning particle with gravimagnetic moment. Comparing our equations to those of spinning particle on electromagnetic background (see (226)-(228)), we see that the two systems have the same structure after the identification $\kappa \sim \mu$ and $\theta_{\mu \nu} \equiv R_{\mu \nu \alpha \beta} S^{\alpha \beta} \sim F_{\mu \nu}$, where $\mu$ is the magnetic moment. That is, a curvature influences trajectory of spinning particle in the same way as the electromagnetic field with the strength $\theta_{\mu \nu}$.

5.2. Ultrarelativistic Limit Requires the Value of Gravimagnetic Moment $\kappa=1$. In the previous subsection, we have formulated Hamiltonian variational problem for spinning particle with gravimagnetic moment $\kappa$ in an arbitrary gravitational background. The model is consistent for any value of $\kappa$. When $\kappa=0$, our equations of motion (149)-(151) coincide with MPTD equations (110). As we have shown above, they have unsatisfactory behavior in ultrarelativistic limit. Consider now our spinning particle with gravimagnetic moment $\kappa=1$. This implies $\widetilde{\mathscr{T}}^{\mu}{ }_{\nu}=\delta^{\mu}{ }_{\nu}$ and $\mathscr{G}_{\mu \nu}=g_{\mu \nu}$ and crucially simplifies the equations of motion. (Besides $S^{\mu \nu} P_{\nu}=0$, there are other supplementary spin conditions [3-7]. In this respect, we point out that the MPTD theory implies this condition with certain $P_{v}$ written in (110). Introducing $\kappa$, we effectively changed $P_{v}$ and hence changed the supplementary spin condition. For instance, when $\kappa=1$ and in the space with $\nabla R=0$, we have $P^{\mu}=(\widetilde{m} c / \sqrt{-\dot{x} g \dot{x}}) \dot{x}^{\mu}$ instead of (110).) Hamiltonian equations (149)-(151) read

$$
\begin{aligned}
\frac{m_{r} c}{\sqrt{-\dot{x} g \dot{x}}} \dot{x}^{\mu} & =P^{\mu}+c Z^{\mu}, \\
\nabla P_{\mu} & =-\frac{1}{4} \theta_{\mu \nu} \dot{x}^{\nu}-\frac{\sqrt{-\dot{x} g \dot{x}}}{32 m_{r} c} \nabla_{\mu}(S \theta), \\
\nabla S^{\mu \nu} & =-\frac{\sqrt{-\dot{x} g \dot{x}}}{4 m_{r} c}(\theta S)^{[\mu \nu]}+2 P^{[\mu} \dot{x}^{\nu]},
\end{aligned}
$$

while the Lagrangian equations are composed now of the equation for trajectory

$$
\nabla\left[\frac{m_{r} \dot{x}^{\mu}}{\sqrt{-\dot{x} g \dot{x}}}\right]=-\frac{1}{4 c} \theta^{\mu}{ }_{\nu} \dot{x}^{\nu}-\frac{\sqrt{-\dot{x} g \dot{x}}}{32 m_{r} c^{2}} \nabla^{\mu}(S \theta)+\nabla Z^{\mu}
$$


and by the equation for precession of spin-tensor:

$$
\nabla S^{\mu \nu}=-\frac{\sqrt{-\dot{x} g \dot{x}}}{4 m_{r} c}(\theta S)^{[\mu \nu]}+2 c \dot{x}^{[\mu} Z^{\nu]} .
$$

These equations can be compared with (92) and (93). In the modified theory, we have the following:

(1) Time interval and distance should be unambiguously defined within the original space-time metric $g_{\mu \nu}$. So, the critical speed is equal to the speed of light.

(2) Covariant precession of spin (160) has a smooth behavior; in particular, for homogeneous field, $\nabla R=$ 0 , we have $\nabla S \sim \sqrt{-\dot{x} g \dot{x}}$ contrary to $\nabla S \sim 1 / \sqrt{-\dot{x} g \dot{x}}$ in (93).

(3) Even in homogeneous field we have modified dynamics for both $x$ and $S$. Equation (159) in the space with homogeneous curvature has the structure similar to (1); hence, we expect that longitudinal acceleration vanishes as $v \rightarrow c$. Let us confirm this by direct computations.

To find the acceleration, we separate derivative of the radiation mass $m_{r}$ and write (159) in the form

$$
\frac{d}{d \tau}\left[\frac{\dot{x}^{\mu}}{\sqrt{-\dot{x} g \dot{x}}}\right]=\frac{f^{\mu}}{\sqrt{-\dot{x} g \dot{x}}}
$$

where the force is

$$
\begin{aligned}
f^{\mu} \equiv & -\Gamma_{\alpha \beta}^{\mu} \dot{x}^{\alpha} \dot{x}^{\beta}-\frac{\sqrt{-\dot{x} g \dot{x}}}{4 m_{r} c} \theta^{\mu}{ }_{\nu} \dot{x}^{\nu}+\frac{\dot{x} g \dot{x}}{32 m_{r}^{2} c^{2}} \nabla^{\mu}(S \theta) \\
& +\frac{\sqrt{-\dot{x} g \dot{x}}}{m_{r}} \nabla Z^{\mu}-\dot{x}^{\mu} \frac{\dot{m}_{r}}{m_{r}} .
\end{aligned}
$$

While this expression contains derivatives of spin due to $\dot{m}_{r}$-term, the resulting expression is nonsingular function of velocity because $\nabla S$ is a smooth function. Hence, contrary to (123), the force now is nonsingular function of velocity. We take $\tau=x^{0}$ in the spacial part of system (161); this gives

$$
\left(\frac{d t}{d x^{0}}\right)^{-1} \frac{d}{d x^{0}}\left[\frac{v^{i}}{\sqrt{c^{2}-(\mathbf{v} \gamma \mathbf{v})}}\right]=\frac{f^{i}(v)}{\sqrt{c^{2}-(\mathbf{v} \gamma \mathbf{v})}}
$$

where $f^{i}(v)$ is obtained from (162) replacing $\dot{x}^{\mu}$ by $v^{\mu}$ of (10). This system is of the form in (29), so the acceleration is given by (38) and (39):

$$
\begin{aligned}
a^{i} & =\widetilde{M}_{j}^{i}\left[f^{j}+\widetilde{\Gamma}^{j}{ }_{k l}(\gamma) v^{k} v^{l}\right]+\frac{1}{2}\left(\frac{d t}{d x^{0}}\right)^{-1} \\
\cdot & {\left[\left(\mathbf{v} \partial_{0} \gamma \gamma^{-1}\right)^{i}-\frac{\left(\mathbf{v} \partial_{0} \gamma \mathbf{v}\right)}{c^{2}} v^{i}\right], } \\
\mathbf{v} \gamma \mathbf{a} & =\left(1-\frac{\mathbf{v} \gamma \mathbf{v}}{c^{2}}\right)\left[(\mathbf{v} \gamma)_{i}\left[f^{i}(v)+\widetilde{\Gamma}_{k l}^{i}(\gamma) v^{k} v^{l}\right]\right. \\
& \left.+\frac{1}{2}\left(\frac{d t}{d x^{0}}\right)^{-1}\left(\mathbf{v} \partial_{0} \gamma \mathbf{v}\right)\right] .
\end{aligned}
$$

With the smooth $f^{i}$ given in (162), and as $v \rightarrow c$, acceleration (164) remains finite while longitudinal acceleration (165) vanishes. Due to identity (36), we have $(\mathbf{v} \gamma)_{i} f^{i} \stackrel{v \rightarrow c}{\longrightarrow}$ $-(\mathbf{v} \gamma)_{i} \Gamma^{i}{ }_{\alpha \beta} \dot{x}^{\alpha} \dot{x}^{\beta}$; that is, the trajectory tends to that of spinless particle in the limit.

In resume, contrary to MPTD equations, the modified theory is consistent with respect to the original metric $g_{\mu \nu}$. Hence, the modified equations could be more promising for description of the rotating objects in astrophysics. It would be interesting to see if the nonminimal interaction allows removing the space-like-time-like transitions observed for spinning particle in the Schwarzschild background [24].

We should note that MPTD equations follow from a particular form assumed for the multipole representation of a rotating body [1]. It would be interesting to find a set of multipoles which yields modified equations (157).

5.3. Lagrangian of Spinning Particle with Gravimagnetic Moment. We look for the Lagrangian which in the phase space implies variational problem (137). First, we note that the constraints $\omega \pi=P \omega=0$ always appear from the Lagrangian which depends on $N \dot{x}$ and $N \dot{\omega}$. So, we omit the corresponding terms in (137). Second, we present the remaining terms in (137) in the form

$$
\begin{aligned}
S_{\kappa} & =\int d \tau p_{\mu} \dot{x}^{\mu}+\pi_{\mu} \dot{\omega}^{\mu}-\frac{\lambda_{1}}{2}(P, \pi)\left(\begin{array}{cc}
g & \lambda g \\
\lambda g & \sigma
\end{array}\right)\left(\begin{array}{l}
P \\
\pi
\end{array}\right) \\
& -\frac{\lambda_{1}}{2}\left[(m c)^{2}-\frac{\alpha}{\omega^{2}}\right],
\end{aligned}
$$

where we have introduced the symmetric matrix

$$
\begin{aligned}
\sigma^{\mu \nu} & =g^{\mu \nu}+\kappa R_{\alpha}{ }^{\mu}{ }_{\beta}^{\nu} \omega^{\alpha} \omega^{\beta}, \\
\text { then } \sigma^{\mu \nu} \omega_{\nu} & =\omega^{\mu} .
\end{aligned}
$$

The matrix which appeared in (166) is invertible; the inverse matrix is

$$
\left(\begin{array}{cc}
K \sigma & -\lambda K \\
-\lambda K & K
\end{array}\right), \quad \text { where } K=\left(\sigma-\lambda^{2} g\right)^{-1}
$$

When $\kappa=0$, we have $K^{\mu \nu}=\left(1-\lambda^{2}\right)^{-1} g^{\mu \nu}$, and (168) coincides with the matrix which appeared in (53). Third, we note that the Hamiltonian variational problem of the form $p \dot{q}-\left(\lambda_{1} / 2\right) p A p$ follows from the reparametrization-invariant Lagrangian $\sqrt{\dot{q} A^{-1} \dot{q}}$. So, we tentatively replace the matrix which appeared in free Lagrangian (53) by (168) and switch on the minimal interaction of spin with gravity, $\dot{\omega} \rightarrow \nabla \omega$. This gives the following Lagrangian formulation of spinning particle with gravimagnetic moment:

$$
\begin{gathered}
L=-\sqrt{(m c)^{2}-\frac{\alpha}{\omega^{2}}} \sqrt{-(N \dot{x}, N \nabla \omega)\left(\begin{array}{cc}
K \sigma & -\lambda K \\
-\lambda K & K
\end{array}\right)\left(\begin{array}{c}
N \dot{x} \\
N \nabla \omega
\end{array}\right)}= \\
-\sqrt{(m c)^{2}-\frac{\alpha}{\omega^{2}}} \sqrt{-\dot{x} N K \sigma N \dot{x}-\nabla \omega N K N \nabla \omega+2 \lambda \dot{x} N K N \nabla \omega} .
\end{gathered}
$$


Let us show that this leads to the desired Hamiltonian formulation (137). The matrixes $\sigma, K$, and $N$ are symmetric and mutually commuting. Canonical momentum for $\lambda$ vanishes and hence represents the primary constraint, $p_{\lambda}=0$. In terms of the canonical momentum $P_{\mu} \equiv p_{\mu}-\Gamma_{\alpha \mu}^{\beta} \omega^{\alpha} \pi_{\beta}$, the expressions for conjugate momenta $p_{\mu}=\partial L / \partial \dot{x}^{\mu}$ and $\pi_{\mu}=\partial L / \partial \dot{\omega}^{\mu} \mathrm{read}$

$$
\begin{aligned}
P_{\mu} & =\frac{1}{L_{0}}\left[m^{2} c^{2}-\frac{\alpha}{\omega^{2}}\right]^{1 / 2} \\
\cdot & {\left[(\dot{x} N K \sigma N)_{\mu}-\lambda(\nabla \omega N K N)_{\mu}\right], } \\
\pi_{\mu}= & \frac{1}{\sqrt{2} L_{0}}\left[m^{2} c^{2}-\frac{\alpha}{\omega^{2}}\right]^{1 / 2} \\
\cdot & {\left[(\nabla \omega N K N)_{\mu}-\lambda(\dot{x} N K N)_{\mu}\right], }
\end{aligned}
$$

where $L_{0}$ is the second square root in (170). They immediately imply the primary constraints $\omega \pi=0$ and $P \omega=0$. From the expressions

$$
\begin{aligned}
P^{2} & =\frac{1}{L_{0}^{2}}\left[(m c)^{2}-\frac{\alpha}{\omega^{2}}\right][(\dot{x} N K \sigma K \sigma N \dot{x}) \\
& \left.+\lambda^{2}(\nabla \omega N K K N \nabla \omega)-2 \lambda(\dot{x} N K \sigma K N \nabla \omega)\right], \\
\pi \sigma \pi & =\frac{1}{L_{0}^{2}}\left[(m c)^{2}-\frac{\alpha}{\omega^{2}}\right]\left[\lambda^{2}(\dot{x} N K \sigma K N \dot{x})\right. \\
& +(\nabla \omega N K \sigma K N \nabla \omega)-2 \lambda(\dot{x} N K \sigma K N \nabla \omega)], \\
2 \lambda P & \pi=\frac{1}{L_{0}^{2}}\left[(m c)^{2}-\frac{\alpha}{\omega^{2}}\right]\left[-2 \lambda^{2}(\dot{x} N K \sigma K N \dot{x})\right. \\
& -2 \lambda^{2}(\nabla \omega N K K N \nabla \omega)+2 \lambda(\dot{x} N K \sigma K N \nabla \omega) \\
& \left.+2 \lambda^{3}(\dot{x} N K K N \nabla \omega)\right],
\end{aligned}
$$

we conclude that their sum does not depend on velocities and hence gives one more constraint:

$$
P^{2}+\pi \sigma \pi+2 \lambda P \pi=-\left[(m c)^{2}-\frac{\alpha}{\omega^{2}}\right] .
$$

Then, the Hamiltonian is $H=P \dot{x}+\pi \nabla \omega-L+\lambda_{i} T_{i}$. From (171), we obtain $P \dot{x}+\pi \nabla \omega=L$, so the Hamiltonian is composed of primary constraints:

$$
\begin{aligned}
H & =\frac{\lambda_{1}}{2}\left[P^{2}+\kappa R_{\alpha \mu \beta \gamma} \omega^{\alpha} \pi^{\mu} \omega^{\beta} \pi^{\nu}+(m c)^{2}+\pi^{2}-\frac{\alpha}{\omega^{2}}\right. \\
& +2 \lambda(P \pi)]+\lambda_{2}(\omega \pi)+\lambda_{3}(P \omega)+v p_{\lambda} .
\end{aligned}
$$

This expression is equivalent to the Hamiltonian written in variational problem (137). Problems (137) and (174) yield the same equations for the set $x^{\mu}, P^{\mu}$, and $S^{\mu \nu}$.

\section{Spinless Particle Nonminimally Interacting with Electromagnetic Field, Speed of Light, and Critical Speed}

In this section, we consider examples of manifestly Poincarecovariant and reparametrization-invariant equations of spinless particle in Minkowski space which lead to critical speed different from the speed of light. We achieve this assuming a nonminimal interaction with electromagnetic field. These toy models confirm that the critical speed different from the speed of light does not contradict relativistic invariance (existence of the observer-independent scale $c$ ).

Let us denote $x^{i}(t), i=1,2,3$, physical dynamical variables describing trajectory of relativistic particle subject to an external force. In order to work with manifestly covariant quantities, we use parametric equations of the trajectory $x^{\mu}(\tau)=\left(x^{0} \equiv \operatorname{ct}(\tau), x^{i}(\tau)\right)$, where $\tau$ is an arbitrary parameter along the world line. In this section, we use the usual special-relativity notions for time, three-dimensional distance, velocity, and acceleration as well as for the scalar product:

$$
\begin{aligned}
& v^{i}=\frac{d x^{i}}{d t}, \\
& a^{i}=\frac{d v^{i}}{d t}, \\
& \mathbf{v a}=v^{i} a^{i} .
\end{aligned}
$$

Let us start from the standard Lagrangian of spinless particle in electromagnetic field. Using the auxiliary variable $\lambda$, the Lagrangian reads

$$
L=\frac{1}{2 \lambda} \dot{x}^{2}-\frac{\lambda}{2} m^{2} c^{2}+\frac{e}{c} A \dot{x} .
$$

This implies the manifestly relativistic equations.

$$
\left(\frac{\dot{x}^{i}}{\sqrt{-\dot{x}^{2}}}\right)=\frac{e}{m c^{2}} F_{\nu}^{\mu} \dot{x}^{\nu} .
$$

They became singular as $\dot{x}^{2} \rightarrow 0$. Using the reparametrization-invariant derivative $D=\left(1 / \sqrt{-\dot{x}^{2}}\right)(d / d \tau)$, they read in manifestly reparametrization-invariant form

$$
D D x^{\mu}=f^{\mu} \equiv \frac{e}{m c^{2}} F^{\mu}{ }_{\nu} D x^{\nu} .
$$

Due to the identities

$$
\begin{aligned}
\dot{x}_{\mu} D D x^{\mu} & =0, \\
\dot{x}_{\mu} f^{\mu} & =0,
\end{aligned}
$$

the system contains only three independent equations. The first identity became more transparent if we compute derivative on the left hand side of (177); then the system reads

$$
M^{\mu}{ }_{\nu} \ddot{x}^{\nu}=\frac{e \sqrt{-\dot{x}^{2}}}{m c^{2}} F^{\mu}{ }_{\nu} \dot{x}^{\nu},
$$


where $M^{\mu}{ }_{\nu}$ turns out to be projector on the plane orthogonal to $\dot{x}^{\mu}$ :

$$
\begin{aligned}
M^{\mu}{ }_{\nu} & =\delta^{\mu}{ }_{\nu}-\frac{\dot{x}^{\mu} \dot{x}_{v}}{\dot{x}^{2}}, \\
\dot{x}_{\mu} M^{\mu}{ }_{\nu} & =0 .
\end{aligned}
$$

Using reparametrization invariance, we can take physical time as the parameter, $\tau=t$; this directly yields equations for observable dynamical variables $x^{i}(t)$. In the physical-time parametrization, we have $x^{\mu}=(c t, \mathbf{x}(t)), \dot{x}^{\mu}=(c, \mathbf{v}(t))$, and $1 / \sqrt{-\dot{x}^{2}}=1 / \sqrt{c^{2}-\mathbf{v}^{2}}$. Time-like component of system (177) reads

$$
\frac{d}{d t} \frac{c}{\sqrt{c^{2}-\mathbf{v}^{2}}}=\frac{e}{m c^{2}} F_{i}^{0} \dot{x}^{i}
$$

and gives the value of acceleration along the direction of velocity:

$$
\mathbf{v a}=\frac{e\left(c^{2}-\mathbf{v}^{2}\right)^{3 / 2}}{m c^{3}}(\mathbf{E v}) .
$$

The longitudinal acceleration vanishes as $|\mathbf{v}| \rightarrow c$. Hence, the singularity in (177) implies that the particles speed can not exceed the value $c$.

Components of three-acceleration vector can be obtained from the spatial part of system (177):

$$
\frac{\ddot{x}^{i}}{\sqrt{c^{2}-\mathbf{v}^{2}}}+\dot{x}^{i} \frac{d}{d t} \frac{1}{\sqrt{c^{2}-\mathbf{v}^{2}}}=\frac{e}{m c^{2}} F^{i} \dot{x}^{\nu} .
$$

Using (182), we obtain

$$
\mathbf{a}=\frac{\sqrt{c^{2}-\mathbf{v}^{2}}}{m c}\left[e \mathbf{E}-\frac{e(\mathbf{E v})}{c^{2}} \mathbf{v}+\frac{e}{c} \mathbf{v} \times \mathbf{B}\right] .
$$

In accordance with degeneracy (179), scalar product of the spacial part with $\mathbf{v}$ gives time-like component (183).

Let us discuss two modifications which preserve both relativistic covariance and reparametrization invariance of equations of motion but could yield nonvanishing longitudinal acceleration as $|\mathbf{v}| \rightarrow c$.

First, in the presence of external fields we can construct an additional reparametrization invariant. For instance, we can use the derivative

$$
D^{\prime} \equiv \frac{1}{\sqrt{-\dot{x} g \dot{x}}} \frac{d}{d \tau},
$$

where the usual relativistic factor $\dot{x} \eta \dot{x}$ is replaced by

$$
\begin{aligned}
-\dot{x}^{\mu} g_{\mu \nu} \dot{x}^{\nu} & =-\dot{x}^{2}-\epsilon k(\dot{x} F F \dot{x}) \\
& =c^{2}-\mathbf{v}^{2}-\epsilon k(\dot{x} F F \dot{x}), \quad \epsilon= \pm 1 .
\end{aligned}
$$

The right dimension can be supplied by the constant $k$ equal to $e^{6} / m^{4} c^{8}$ or $\hbar^{3} / m^{4} c^{5}$. Second, we consider nonminimal interaction with the force constructed from reparametrization-invariant quantities, $f^{\mu}\left(D^{\prime} x, F, \partial F, \ldots\right)=$ $f^{\mu}{ }_{0}+f^{\mu}{ }_{v} D^{\prime} x^{\nu}+\cdots$. Hence, let us consider the manifestly covariant and reparametrization-invariant equations

$$
\begin{aligned}
D^{\prime} D^{\prime} x^{\mu} & =f^{\mu} \\
\text { or } M^{\mu}{ }_{\nu} \ddot{x}^{\nu} & =-(\dot{x} g \dot{x}) f^{\mu}+\frac{(\dot{x} \dot{g} \dot{x})}{2(\dot{x} g \dot{x})} \dot{x}^{\mu},
\end{aligned}
$$

where

$$
\begin{aligned}
M^{\mu}{ }_{\nu} & =\delta^{\mu}{ }_{v}-\frac{\dot{x}^{\mu}(\dot{x} g)_{v}}{(\dot{x} g \dot{x})}, \\
(\dot{x} g)_{\mu} M^{\mu}{ }_{\nu} & =0 \\
M^{\mu}{ }_{v} \dot{x}^{\nu} & =0 .
\end{aligned}
$$

Due to noninvertibility of $M^{\mu}{ }_{v}$, system (188) consists of three second-order equations and one equation of first order. Contracting (188) with $(\dot{x} g)_{\mu}$, we obtain the first-order equation

$$
\dot{x} \dot{g} \dot{x}=2(\dot{x} g \dot{x})(\dot{x} g f) \text {. }
$$

This is analog of $\dot{x}_{\mu} f^{\mu}=0$ of the previous case in (179) and can be used to present $f^{0}$ through three-dimensional force. Using reparametrization invariance, we take $\tau=x^{0}$ and write (188) in the form

$$
\begin{aligned}
& \frac{d}{d x^{0}} \frac{1}{\sqrt{-\dot{x} g \dot{x}}}=\sqrt{-\dot{x} g \dot{x}} f^{0}, \\
& \frac{\ddot{x}^{i}}{\sqrt{-\dot{x} g \dot{x}}}+\dot{x}^{i} \frac{d}{d x^{0}} \frac{1}{\sqrt{-\dot{x} g \dot{x}}}=\sqrt{-\dot{x} g \dot{x}} f^{i} \text {. }
\end{aligned}
$$

Using (191) in (192), we obtain

$$
\begin{aligned}
\frac{d^{2} x^{i}}{d x^{02}} & =-\dot{x} g \dot{x}\left[f^{i}-f^{0} \frac{d x^{i}}{d x^{0}}\right], \\
\dot{x}^{\mu} & =\frac{d x^{\mu}}{d x^{0}} .
\end{aligned}
$$

We take $x^{0}=c t$ and then three-acceleration

$$
\begin{aligned}
\mathbf{a} & =-\dot{x} g \dot{x}\left[\mathbf{f}-\frac{f^{0}}{c} \mathbf{v}\right], \\
\dot{x}^{\mu} & =\left(c, \mathbf{v}=\frac{d \mathbf{x}}{d t}\right) .
\end{aligned}
$$

Equations (190) and (194) are equivalent to initial system (188). Equation (194) implies the longitudinal acceleration:

$$
\begin{aligned}
\mathbf{v a} & =-\dot{x} g \dot{x}\left[\mathbf{v f}-\frac{\mathbf{v}^{2}}{c} f^{0}\right] \\
& \equiv-\dot{x} g \dot{x}\left[\frac{c^{2}-\mathbf{v}^{2}}{c} f^{0}+\dot{x}_{\mu} f^{\mu}\right], \\
f^{\mu} & =f^{\mu}\left(\frac{\dot{x}}{\sqrt{-\dot{x} g \dot{x}}}, F, \partial F, \ldots\right) .
\end{aligned}
$$


The acceleration vanishes at the values of speed which zero out r.h.s. of this equation. If in physical-time parametrization the four-force obeys the identity $\dot{x}_{\mu} f^{\mu}=0$, we have two special points, $|\mathbf{v}|=c$ and $v^{\prime}$, determined from $\dot{x} g \dot{x}=0$. In absence of the identity, and if $\dot{x}_{\mu} f^{\mu} \neq 0$ as $|\mathbf{v}| \rightarrow c$, the speed of light does not represent a special point of (195). Let us illustrate this equation with two examples.

Example 1. Consider the minimal interaction $f^{\mu}=$ $\left(e / m c^{2}\right) F^{\mu}{ }_{\nu} D^{\prime} x^{\nu}$ and relativistic-contraction factor (187); then (195) reads

$$
\mathbf{v a}=\frac{e(\mathbf{v E})}{m c^{3}} \sqrt{-\dot{x} g \dot{x}}\left(c^{2}-\mathbf{v}^{2}\right) .
$$

Besides the usual special point, $\mathbf{v}^{2}=c^{2}$, there is one more, say $v^{\prime}=\left|\mathbf{v}^{\prime}\right|$, determined by $\dot{x} g \dot{x}=0$. This surface is slightly different from the sphere $c^{2}-\mathbf{v}^{2}=0$. So, the second special point generally differs from the speed of light. To see this, we compute the last term in (187):

$$
-\dot{x} F F \dot{x}=c^{2} E_{i}\left(\delta_{i j}-\frac{v_{i} v_{j}}{c^{2}}\right) E_{j}+\mathbf{v}^{2} B_{i} N_{i j} B_{j} .
$$

Here, $N_{i j} \equiv \delta_{i j}-v_{i} v_{j} / \mathbf{v}^{2}$ is projection operator on the plane orthogonal to the vector $\mathbf{v}$, so we can write $\mathbf{B} N \mathbf{B}=(N \mathbf{B})^{2}=$ $\mathbf{B}_{\perp}^{2}$. Then, factor (187) reads

$$
-\dot{x} g \dot{x}=c^{2}-\mathbf{v}^{2}+\epsilon k\left[c^{2} \mathbf{E}\left(1-\frac{\mathbf{v}}{c^{2}}\right) \mathbf{E}+\mathbf{v}^{2} \mathbf{B}_{\perp}^{2}\right] .
$$

The quantity $\delta_{i j}-v_{i} v_{j} / c^{2}$ turns into the projection operator $N$ when $|\mathbf{v}|=c$. Hence,

$$
-\dot{x} g \dot{x} \stackrel{|\mathbf{v}| \rightarrow c}{\longrightarrow} \epsilon k c^{2}\left[\mathbf{E}_{\perp}^{2}+\mathbf{B}_{\perp}^{2}\right]
$$

If $\mathbf{E}$ and $\mathbf{B}$ are not mutually parallel in the laboratory system, this expression does not vanish for any orientation of $\mathbf{v}$. This implies that factor (187) does not vanish at $|\mathbf{v}|=c$.

We confirmed that longitudinal acceleration generally vanishes at two different values of speed, $c$ and $v^{\prime}$. Then, (196) implies the following possibilities.

(A) Let $\epsilon=+1$; then from (198) we conclude $c<v^{\prime}$, and speed of the particle approximates to $c$. The second special point $v^{\prime}$ turns out to be irrelevant. So, $v_{\mathrm{cr}}=c$.

(B) Let $\epsilon=-1$; then $v^{\prime}<c$, and the particle with small initial velocity will approximate to the critical velocity $v_{\mathrm{cr}}=v^{\prime}<c$. So, it never approximates to the speed of light.

Example 2 (possibility of superluminal motion?). Take relativistic-contraction factor (187) with $\epsilon=+1$ and nonparallel electric and magnetic fields. As we have seen above, this implies $c<v^{\prime}$, where $v^{\prime}$ is a solution of $\dot{x} g \dot{x}=0$. Consider the nonminimal interaction

$$
\begin{gathered}
f^{\mu}=\frac{e}{m c^{2}} F^{\mu}{ }_{\nu} D^{\prime} x^{\nu}+\widetilde{f}^{\mu}, \\
\text { where } \widetilde{f}^{\mu}=-\widetilde{k}^{2} D^{\prime} x^{\alpha} \partial^{\mu}(F F)_{\alpha \beta} D^{\prime} x^{\beta} .
\end{gathered}
$$

We assume homogeneous and nonstationary fields with growing tension:

$$
\begin{aligned}
\partial^{i} \mathbf{E} & =\partial^{i} \mathbf{B}=0, \\
\frac{d}{d t}|\mathbf{E}| & >0, \\
\frac{d}{d t}|\mathbf{B}| & >0 ;
\end{aligned}
$$

then $\widetilde{f}^{i}=0, \widetilde{f}^{0}=-\left(\widetilde{k}^{2} / c \dot{x} g \dot{x}\right) \dot{x}(\partial / \partial t)(F F) \dot{x}$. The longitudinal acceleration reads

$$
\begin{aligned}
\mathbf{v a} & =a_{1}(v)+a_{2}(v) \\
& \equiv \frac{e(\mathbf{v E})}{m c^{3}} \sqrt{-\dot{x} g \dot{x}}\left(c^{2}-\mathbf{v}^{2}\right)-\frac{\tilde{k}^{2} \mathbf{v}^{2}}{c^{2}} \dot{x} \frac{\partial}{\partial t}(F F) \dot{x} .
\end{aligned}
$$

We have $a_{1}(c)=0$, while $a_{2}(c)$ is positive according to (197) and (201). So, the particle overcomes the light barrier. In the region $c<v<v^{\prime}$, we have $a_{1}(v)<0$ and $a_{2}(v)>0$, so the particle will continue to accelerate up to critical velocity $v_{\mathrm{cr}}$ determined by the equation $a_{1}+a_{2}=0$. If $a_{2}>\left|a_{1}\right|$ in the region, the particle will accelerate up to the value $v_{\mathrm{cr}}=v^{\prime}$. Above this velocity, (202) becomes meaningless.

The toy examples show that critical speed in a manifestly relativistic and reparametrization-invariant theory does not always coincide with the speed of light, if we assume the usual special-relativity definitions of time and distance. In general case, we expect that $\mathbf{v}_{\mathrm{cr}}$ is both field- and spin-dependent quantity. In the next section, we repeat this analysis for more realistic case of a particle with spin.

\section{Spinning Particle in an Arbitrary Electromagnetic Field}

7.1. Lagrangian and Hamiltonian Formulations. In formulation (51), the vector model of spin admits interaction with an arbitrary electromagnetic field. To introduce coupling of the position variable $x$ with electromagnetic field, we add the minimal interaction $(e / c) A_{\mu} \dot{x}^{\mu}$. As for spin, it couples with $A^{\mu}$ through the term

$$
D \omega^{\mu} \equiv \dot{\omega}^{\mu}-\lambda \frac{e \mu}{c} F^{\mu \nu} \omega_{\nu}
$$

where the coupling constant $\mu$ is the magnetic moment. They are the only terms we have found compatible with symmetries and constraints which should be presented in the theory. Adding these terms to the free theory in (51), we obtain the action

$$
\begin{aligned}
S & =\int d \tau \frac{1}{4 \lambda}[\dot{x} N \dot{x}+D \omega N D \omega \\
& \left.-\sqrt{[\dot{x} N \dot{x}+D \omega N D \omega]^{2}-4(\dot{x} N D \omega)^{2}}\right] \\
& -\frac{\lambda}{2}\left(m^{2} c^{2}-\frac{\alpha}{\omega^{2}}\right)+\frac{e}{c} A \dot{x} .
\end{aligned}
$$


In their work [22], Hanson and Regge analyzed whether the spin-tensor in (50) interacts directly with an electromagnetic field and concluded on impossibility to construct the interaction in closed form. In our model, an electromagnetic field interacts with the part $\omega^{\mu}$ of the spin-tensor.

Let us construct Hamiltonian formulation of the model. The procedure which leads to the Hamiltonian turns out to be very similar to that described in Section 4.1, so we present only the final expression (for details, see [32]). Conjugate momenta for $x^{\mu}, \omega^{\mu}$, and $\lambda$ are denoted as $p^{\mu}$, $\pi^{\mu}$, and $p_{\lambda}$. We use also the canonical momentum $\mathscr{P}^{\mu} \equiv$ $p^{\mu}-(e / c) A^{\mu}$. Contrary to $p^{\mu}$, the canonical momentum $\mathscr{P}^{\mu}$ is $U(1)$ gauge-invariant quantity. With these notations, we obtain the Hamiltonian variational problem which is equivalent to (204):

$$
\begin{aligned}
S_{H} & =\int d \tau p_{\mu} \dot{x}^{\mu}+\pi_{\mu} \dot{\omega}^{\mu}+p_{\lambda} \dot{\lambda} \\
& -\left[\frac{\lambda}{2}\left(P^{2}-\frac{e \mu}{2 c}(F S)+(m c)^{2}+\pi^{2}-\frac{\alpha}{\omega^{2}}\right)\right. \\
& \left.+\lambda_{2}(\omega \pi)+\lambda_{3}(P \omega)+\lambda_{4}(P \pi)+\lambda_{0} p_{\lambda}\right] .
\end{aligned}
$$

The expression in square brackets represents the Hamiltonian. By $\lambda_{2}, \lambda_{3}, \lambda_{4}$, and $\lambda_{0}$, we denoted the Lagrangian multipliers; they are written in front of the corresponding primary constraints. The fundamental Poisson brackets $\left\{x^{\mu}, p^{\nu}\right\}=\eta^{\mu \nu}$ and $\left\{\omega^{\mu}, \pi^{\nu}\right\}=\eta^{\mu \nu}$ imply

$$
\begin{aligned}
\left\{x^{\mu}, \mathscr{P}^{\nu}\right\} & =\eta^{\mu \nu}, \\
\left\{\mathscr{P}^{\mu}, \mathscr{P}^{\nu}\right\} & =\frac{e}{c} F^{\mu \nu}, \\
\left\{S^{\mu \nu}, S^{\alpha \beta}\right\} & =2\left(\eta^{\mu \alpha} S^{\nu \beta}-\eta^{\mu \beta} S^{\nu \alpha}-\eta^{\nu \alpha} S^{\mu \beta}+\eta^{\nu \beta} S^{\mu \alpha}\right) .
\end{aligned}
$$

According to (207), the spin-tensor is generator of Lorentz algebra $S O(1,3)$. As $\omega \pi, \omega^{2}$, and $\pi^{2}$ are Lorentz-invariants, they have vanishing Poisson brackets with $S^{\mu \nu}$. To reveal the higher-stage constraints, we write the equations $\dot{T}_{i}=$ $\left\{T_{i}, H\right\}=0$. The Dirac procedure stops on third stage with the following equations:

$$
\begin{gathered}
p_{\lambda}=0 \Longrightarrow \\
T_{1} \equiv \mathscr{P}^{2}-\frac{e \mu}{2 c}(F S)+m^{2} c^{2}+\pi^{2}-\frac{\alpha}{\omega^{2}}=0 \Longrightarrow \\
\lambda_{3} C+\lambda_{4} D=0 \\
T_{2} \equiv(\omega \pi)=0 \Longrightarrow T_{5} \equiv \pi^{2}-\frac{\alpha}{\omega^{2}}=0, \\
T_{3} \equiv(\mathscr{P} \omega)=0 \Longrightarrow \lambda_{4}=-\frac{2 \lambda c}{e} a C, \\
T_{4} \equiv(\mathscr{P} \pi)=0 \Longrightarrow \lambda_{3}=\frac{2 \lambda c}{e} a D .
\end{gathered}
$$

We have denoted

$$
\begin{aligned}
& C=-\frac{e(\mu-1)}{c}(\omega F \mathscr{P})+\frac{e \mu}{4 c}(\omega \partial)(F S), \\
& D=-\frac{e(\mu-1)}{c}(\pi F \mathscr{P})+\frac{e \mu}{4 c}(\pi \partial)(F S) .
\end{aligned}
$$

Besides, here and below we will use the following notation. In the equation which relates velocity and canonical momentum will appear the matrix $T^{\mu \nu}$ :

$$
\begin{aligned}
T^{\mu \nu} & =\eta^{\mu \nu}-(\mu-1) a(S F)^{\mu \nu} \\
a & =\frac{-2 e}{4 m^{2} c^{3}-e(2 \mu+1)(S F)} .
\end{aligned}
$$

Using the identity $S^{\mu \alpha} F_{\alpha \beta} S^{\beta \nu}=-(1 / 2)\left(S^{\alpha \beta} F_{\alpha \beta}\right) S^{\mu \nu}$, we find the inverse matrix

$$
\begin{aligned}
\widetilde{\mathscr{T}}^{\mu \nu} & =\eta^{\mu \nu}+(\mu-1) b(S F)^{\mu \nu} \\
b & =\frac{2 a}{2+(\mu-1) a(S F)} \equiv \frac{-2 e}{4 m^{2} c^{3}-3 e \mu(S F)} .
\end{aligned}
$$

The vector $Z^{\mu}$ is defined by

$$
Z^{\mu}=\frac{b}{4 c} S^{\mu \sigma}\left(\partial_{\sigma} F_{\alpha \beta}\right) S^{\alpha \beta} \equiv \frac{b}{4 c} S^{\mu \sigma} \partial_{\sigma}(F S)
$$

This vanishes for homogeneous field; $\partial F=0$. The last equation from (208) turns out to be a consequence of (210) and (211) and can be omitted. Due to the secondary constraint, $T_{5}$ appeared in (209); we can replace the constraint $T_{1}$ on the equivalent one:

$$
T_{1} \equiv \mathscr{P}^{2}-\frac{e \mu}{2 c}(F S)+m^{2} c^{2}=0
$$

The Dirac procedure revealed two secondary constraints written in (216) and (209) and fixed the Lagrangian multipliers $\lambda_{3}$ and $\lambda_{4}$; the latter can be substituted into the Hamiltonian. The multipliers $\lambda_{0}$ and $\lambda_{2}$ and the auxiliary variable $\lambda$ have not been determined. $H$ vanishes on the complete constraint surface, as it should be in a reparametrizationinvariant theory.

We summarized the algebra of Poisson brackets between constraints in Table 2 . The constraints $p_{\lambda}, T_{1}, T_{2}$, and $T_{5}$ form the first-class subset, while $T_{3}$ and $T_{4}$ represent a pair of second class. The presence of two primary first-class constraints $p_{\lambda}$ and $T_{2}$ is in correspondence with the fact that two Lagrangian multipliers remain undetermined within the Dirac procedure.

The evolution of the basic variables was obtained according the standard rule $\dot{z}=\{z, H\}$ (equivalently, we can look for 
TABLE 2: Algebra of constraints.

\begin{tabular}{lccccc}
\hline & $T_{1}$ & $T_{5}$ & $T_{2}$ & $T_{3}$ & $T_{4}$ \\
\hline$T_{1}=\mathscr{P}^{2}-\frac{\mu e}{2 c}(F S)+m^{2} c^{2}$ & 0 & 0 & 0 & $-2 C$ & $-2 D$ \\
$T_{5}=\pi^{2}-\frac{\alpha}{\omega^{2}}$ & 0 & 0 & $-2 T_{5} \approx 0$ & $-2 T_{4} \approx 0$ & $2 \alpha$ \\
$T_{2}=\omega \pi$ & 0 & $2 T_{5} \approx 0$ & 0 & $-T_{3} \approx 0$ & 0 \\
$T_{3}=\mathscr{P} \omega$ & $2 C$ & $2 T_{4} \approx 0$ & $T_{3} \approx 0$ & $-T_{3} \approx 0$ & $T_{4} \approx 0$ \\
$T_{4}=\mathscr{P}_{\pi}$ & $2 D$ & $-\frac{2 \alpha}{\left(\omega^{2}\right)^{2}} T_{3} \approx 0$ & $-T_{4} \approx 0$ & $-T_{1}-\frac{e}{2 c a} \approx-\frac{e}{2 c a}$ & $T_{1}+\frac{e}{2 c a} \approx \frac{e}{2 c a}$ \\
\hline
\end{tabular}

the extremum of variational problem (205)). The equations read

$$
\begin{aligned}
\dot{x}^{\mu} & =\lambda\left(T^{\mu}{ }_{\nu^{\prime}}+\frac{\mu c a}{b} Z^{\mu}\right), \\
\dot{\mathscr{P}}^{\mu} & =\frac{e}{c}(F \dot{x})^{\mu}+\lambda \frac{\mu e}{4 c} \partial^{\mu}(F S), \\
\dot{\omega}^{\mu} & =\lambda \frac{e \mu}{c}(F \omega)^{\mu}-\lambda \frac{2 c a C}{e} \mathscr{P}^{\mu}+\pi^{\mu}+\lambda_{2} \omega^{\mu}, \\
\dot{\pi}^{\mu} & =\lambda \frac{e \mu}{c}(F \pi)^{\mu}-\lambda \frac{2 c a D}{e} \mathscr{P}^{\mu}-\frac{\alpha}{\left(\omega^{2}\right)^{2}} \omega^{\mu}-\lambda_{5} 2 \pi^{\mu} .
\end{aligned}
$$

The ambiguity due to the variables $\lambda$ and $\lambda_{2}$ means that the interacting theory preserves both reparametrization and spin-plane symmetries of the free theory. As a consequence, all the basic variables have ambiguous evolution. $x^{\mu}$ and $\mathscr{P}^{\mu}$ have one-parametric ambiguity due to $\lambda$ while $\omega$ and $\pi$ have two-parametric ambiguity due to $\lambda$ and $\lambda_{2}$. The variables with ambiguous dynamics do not represent observable quantities, so we look for the variables that can be candidates for observable quantities. We note that (218) implies an equation for $S^{\mu \nu}$ which does not contain $\lambda_{2}$ :

$$
\dot{S}^{\mu \nu}=\lambda \frac{e \mu}{c}(F S)^{[\mu \nu]}+2 \mathscr{P}^{[\mu} \dot{x}^{\nu]} .
$$

This proves that the spin-tensor is invariant under local spinplane symmetry. The remaining ambiguity due to $\lambda$ contained in (217) and (219) is related with reparametrization invariance and disappears when we work with physical dynamical variables $x^{i}(t)$. Thus, we will work with $x^{\mu}, \mathscr{P}^{\mu}$, and $S^{\mu \nu}$.

The term $\alpha / 2 \omega^{2}$ in Lagrangian (204) provides the constraint $T_{5}$ which can be written as follows: $\omega^{2} \pi^{2}=\alpha$. Together with $\omega \pi=0$, this implies fixed value of spin:

$$
S^{\mu \nu} S_{\mu \nu}=8\left(\omega^{2} \pi^{2}-(\omega \pi)^{2}\right)=8 \alpha,
$$

for any solution to the equations of motion. The constraints $\omega \mathscr{P}=\pi \mathscr{P}=0$ imply the Pirani condition for the spin-tensor in $(50)$ :

$$
S^{\mu \nu} \mathscr{P}_{v}=0 .
$$

Equations (220) and (221) imply that only two components of spin-tensor are independent, as it should be for spin one-half particle.
Equations (217) and (219), together with (220) and (221), form a closed system which determines evolution of a spinning particle.

The quantities $x^{\mu}, P^{\mu}$, and $S^{\mu \nu}$, being invariant under spin-plane symmetry, have vanishing brackets with the corresponding first-class constraints $T_{2}$ and $T_{5}$. So, obtaining equations for these quantities, we can omit the corresponding terms in Hamiltonian (205). Further, we can construct the Dirac bracket for the second-class pair $T_{3}$ and $T_{4}$. Since the Dirac bracket of a second-class constraint with any quantity vanishes, we can now omit $T_{3}$ and $T_{4}$ from (205). Then, the relativistic Hamiltonian acquires an expected form:

$$
H=\frac{\lambda}{2}\left(\mathscr{P}^{2}-\frac{e \mu}{2 c}(F S)+m^{2} c^{2}\right) .
$$

Equations (217) and (219) follow from this $H$ with use of Dirac bracket, $\dot{z}=\{z, H\}_{\mathrm{DB}}$.

We can exclude the momenta $\mathscr{P}$ and the auxiliary variable $\lambda$ from the equations of motion. This yields second-order equation for the particle's position. To achieve this, we solve the first equation from (217) with respect to $\mathscr{P}$ and use the identities $(S F Z)^{\mu}=-(1 / 2)(S F) Z^{\mu}$ and $\widetilde{\mathscr{T}}^{\mu}{ }_{v} Z^{\nu}=(b / a) Z^{\mu}$; this gives $\mathscr{P}^{\mu}=(1 / \lambda) \widetilde{\mathscr{T}}^{\mu}{ }_{\nu} \dot{x}^{\nu}-\mu c Z^{\mu}$. Then, the Pirani condition reads $(1 / \lambda)(S \widetilde{\mathscr{T}} \dot{x})^{\mu}=\mu c(S Z)^{\mu}$. Using this equality, $\mathscr{P}^{2}$ can be presented as $\mathscr{P}^{2}=\left(1 / \lambda^{2}\right)(\dot{x} G \dot{x})+\mu^{2} c^{2} Z^{2}$, where the symmetric matrix appeared:

$$
\begin{aligned}
G_{\mu \nu} & =\left(\widetilde{\mathscr{T}}^{T} \widetilde{\mathscr{T}}\right)_{\mu \nu} \\
& =\left[\eta+b(\mu-1)(S F+F S)+b^{2}(\mu-1)^{2} F S S F\right]_{\mu \nu} .
\end{aligned}
$$

The matrix $G$ is composed of the Minkowsky metric $\eta_{\mu \nu}$ plus (spin- and field-dependent) contribution; $G_{\mu \nu}=\eta_{\mu \nu}+h_{\mu \nu}(S)$. So, we call $G$ the effective metric produced along the world line by interaction of spin with electromagnetic field. We substitute $\mathscr{P}^{2}$ into constraint (216); this gives expression for $\lambda$ :

$$
\begin{aligned}
\lambda & =\frac{\sqrt{-\dot{x} G \dot{x}}}{m_{r} c}, \\
m_{r}^{2} & =m^{2}-\frac{\mu e}{2 c^{3}}(F S)-\mu^{2} Z^{2} .
\end{aligned}
$$

This shows that the presence of $\lambda$ in (203) implies highly nonlinear interaction of spinning particle with electromagnetic 
field. The final expression of canonical momentum through velocity is

$$
\mathscr{P}^{\mu}=\frac{m_{r} c}{\sqrt{-\dot{x} G \dot{x}}} \widetilde{\mathscr{T}}_{\nu}^{\mu} \dot{x}^{\nu}-\mu c Z^{\mu}
$$

Using (224) and (225), we exclude $\mathscr{P}^{\mu}$ and $\lambda$ from Hamiltonian equations (217), (219), and (221). This gives closed system of Lagrangian equations for the set $x, S$. We have the dynamical equations

$$
\begin{aligned}
D\left[m_{r}(\widetilde{\mathscr{T}} D x)^{\mu}\right]= & \frac{e}{c^{2}}(F D x)^{\mu}+\frac{\mu e}{4 m_{r} c^{3}} \partial^{\mu}(S F) \\
& +\mu D Z^{\mu}, \\
D S^{\mu \nu}= & \frac{e \mu}{m_{r} c^{2}}(F S)^{[\mu \nu]} \\
& -2 b m_{r} c(\mu-1) D x^{[\mu}(S F D x)^{\nu]} \\
& +2 \mu c D x^{[\mu} Z^{\nu]},
\end{aligned}
$$

the Lagrangian counterpart of Pirani condition

$$
S^{\mu \nu}\left[(\widetilde{\mathscr{T}} \dot{x})_{\nu}-\frac{\mu \sqrt{-\dot{x} G \dot{x}}}{m_{r}} Z_{v}\right]=0,
$$

as well as to the value-of-spin condition; $S^{\mu \nu} S_{\mu \nu}=8 \alpha$. In the approximation $O^{3}(S, F, \partial F)$ and when $\mu=1$, they coincide with Frenkel equations; see [31].

Equation (226) shows how spin modifies Lorentz-force equation (1). In general case, the Lorentz force is modified due to the presence of (time-dependent) radiation mass $m_{r}$ (224), the tetrad field $\widetilde{\mathscr{T}}$, and the effective metric $G$ and due to two extra terms on right hand side of (226). Contribution of anomalous magnetic moment $\mu \neq 1$ to the difference between $\dot{x}^{\mu}$ and $\mathscr{P}^{\mu}$ in (225) is proportional to $J / c^{3} \sim \hbar / c^{3}$, while the term with a gradient of field is proportional to $J^{2} / c^{3} \sim \hbar^{2} / c^{3}$.

Consider the homogeneous field;

$$
\begin{aligned}
\partial_{\alpha} F^{\mu \nu} & =0, \\
Z^{\mu} & =0 .
\end{aligned}
$$

Then, contraction of (228) with $F_{\mu \nu}$ yields $(S F)^{\circ}=0$; that is, $S^{\mu \nu} F_{\mu \nu}$ turns out to be the conserved quantity. This implies $\dot{m}_{r}=\dot{a}=\dot{b}=0$. Hence, the Lorentz force is modified due to the presence of time-independent radiation mass $m_{r}$, the tetrad field $\widetilde{\mathscr{T}}$, and the effective metric $G$.

Consider the "classical" value of magnetic moment $\mu=1$. Then, $\widetilde{\mathscr{T}}^{\mu \nu}=\eta^{\mu \nu}$ and $G_{\mu \nu}=\eta_{\mu \nu}$. The Lorentz force is modified due to the presence of time-dependent radiation mass $m_{r}$ and two extra terms on right hand side of (226).

Let us specify the equation for spin precession to the case of uniform and stationary field, supposing also $\mu=1$ and taking physical time as the parameter; $\tau=t$. Then, (228) reduces to the Frenkel condition, $S^{\mu \nu} \dot{x}_{v}=0$, while (227) reads $\dot{S}^{\mu \nu}=\left(e \sqrt{-\dot{x}^{2}} / m_{r} c^{2}\right)(F S)^{[\mu \nu]}$. We decompose spintensor on electric dipole moment $\vec{D}$ and Frenkel spin-vector
$\vec{S}$ according to (50); then $\vec{D}=-(2 / c) \vec{S} \times \vec{v}$, while precession of $\vec{S}$ is given by

$$
\frac{d \vec{S}}{d t}=\frac{e \sqrt{c^{2}-\vec{v}^{2}}}{m_{r} c^{3}}[-\vec{E} \times(\vec{v} \times \vec{S})+c \vec{S} \times \vec{B}]
$$

7.2. Ultrarelativistic Limit within the Usual Special-Relativity Notions. After identification of $\theta_{\mu \nu} \equiv R_{\mu \nu \alpha \beta} S^{\alpha \beta} \sim F_{\mu \nu}$ and $\kappa \sim \mu$, equations of motion in electromagnetic and in gravitational fields acquire the similar structure. Equations (217) and (219) can be compared with (149)-(151) and (226)(227) with (157). In particular, in the Lagrangian equations with anomalous magnetic moment $(\mu \neq 1)$ in Minkowski space also appeared effective metric (223). So, we need to examine the ultrarelativistic limit. In this section, we do this under the usual special-relativity notions; that is, we suppose that the particle probes three-dimensional geometry (175). We show that the critical speed turns out to be different from the speed of light while an acceleration, contrary to Section 4.4, vanishes in ultrarelativistic limit. It will be sufficient to estimate the acceleration in uniform and stationary field (229). We take $\tau=t$ in (226)-(228) and compute the time derivative on 1.h.s. of (226) with $\mu=1,2,3$. Then, the equations read

$$
\begin{aligned}
& a^{i}-\frac{v^{i}}{2(-v G v)} \frac{d}{d t}(-v G v) \\
& =T_{v}^{i}\left[\frac{e \sqrt{-v G v}}{m_{r} c^{2}}(F v)^{i}-\frac{d}{d t} \widetilde{\mathscr{T}}_{\alpha}^{v} v^{\alpha}\right], \\
& \frac{d}{d t} S^{\mu \nu} \\
& =\frac{e \mu \sqrt{-v G v}}{m_{r} c^{2}}(F S)^{[\mu v]} \\
& \quad-\frac{2 b m_{r} c(\mu-1)}{\sqrt{-v G v}} v^{[\mu}(S F v)^{\nu]}, \\
& (S v)^{\mu}+b(\mu-1)(S S F v)^{\mu}=0,
\end{aligned}
$$

where $v^{\mu}=(c, \mathbf{v})$. Equations (233) and (223) imply

$$
-v G v=-v \widetilde{\mathscr{T}} v=c^{2}-\mathbf{v}^{2}-(\mu-1) b(v S F v)
$$

We compute the time derivatives in (231):

$$
\begin{aligned}
& \frac{d}{d t}(-v G v)=-2(\mathbf{v a})-(\mu-1) b\left\{[v(F S+S F)]_{i} a^{i}\right. \\
& \quad+\frac{e \mu \sqrt{-v G v}}{m_{r} c^{2}}[(v F F S v)+(v F S F v)] \\
& \left.\quad-\frac{2 b m_{r} c(\mu-1)}{\sqrt{-v g v}}\left[v^{2}(v F S F v)-(v S F v)(v F v)\right]\right\},
\end{aligned}
$$




$$
\begin{gathered}
-T^{i}{ }_{\nu} \frac{d}{d t} \widetilde{T}_{\alpha}{ }_{\alpha} v^{\alpha}=-\frac{e \sqrt{-v G v}}{m_{r} c^{2}}\left\{\mu(\mu-1) b(F S F v)^{i}\right. \\
\left.-\mu(\mu-1) a(S F F v)^{i}-\mu(\mu-1)^{2} a b(S F F S F v)^{i}\right\} \\
+\frac{2 b m_{r} c(\mu-1)}{\sqrt{-v G v}} T^{i}{ }_{\nu}\left[v^{v}(v F S F v)-(S F v)^{v}(v F v)\right] .
\end{gathered}
$$

We note that all the potentially divergent terms (two last terms in (235) and in (236)), arising due to the contribution from $\dot{S} \sim 1 / \sqrt{-v G v}$, disappear on the symmetry grounds. We substitute nonvanishing terms into (231) obtaining the expression

$$
\begin{aligned}
& M^{i}{ }_{j} a^{j}=\frac{e \sqrt{-v G v}}{m_{r} c^{2}}\left\{(F v)^{i}-\mu(\mu-1) b(F S F v)^{i}\right. \\
& +(\mu-1)^{2} a(S F F[\eta+\mu b S F] v)^{i} \\
& \left.\quad-v^{i} \frac{\mu(\mu-1) b}{2(-v G v)}(v F F S v)\right\},
\end{aligned}
$$

where the matrix

$$
\begin{aligned}
M^{i}{ }_{j}=\delta^{i}{ }_{j}+ & \frac{v^{i} v^{\mu} \Omega_{\mu j}}{2(-v G v)}, \\
& \text { with } \Omega_{\mu j}=2 \delta_{\mu j}+(\mu-1) b(F S+S F)_{\mu j}
\end{aligned}
$$

has the inverse

$$
\widetilde{M}_{j}^{i}=\delta_{j}^{i}-\frac{v^{i} v^{\mu} \Omega_{\mu j}}{2 c^{2}-(\mu-1) b v^{\mu}(F S+S F)_{\mu 0} v^{0}},
$$

with the property

$$
\widetilde{M}_{j}^{i} v^{j}=v^{i} \frac{2(-v G v)}{2 c^{2}-(\mu-1) b v^{\mu}(F S+S F)_{\mu 0} v^{0}} .
$$

Applying the inverse matrix, we obtain the acceleration

$$
\begin{aligned}
a^{i} & =\frac{e \sqrt{-v G v}}{m_{r} c^{2}}\left\{\widetilde { M } _ { j } ^ { i } \left[(F v)^{j}-\mu(\mu-1) b(F S F v)^{j}\right.\right. \\
& \left.+(\mu-1)^{2} a(S F F[\eta+\mu b S F] v)^{j}\right]-v^{i} \\
& \left.\cdot \frac{\mu(\mu-1) b(v F F S v)}{2 c^{2}-(\mu-1) b v^{\mu}(F S+S F)_{\mu 0} v^{0}}\right\} .
\end{aligned}
$$

For the particle with nonanomalous magnetic moment $(\mu=$ 1 ), the right hand side reduces to the Lorentz force, so the expression in braces is certainly nonvanishing in the ultrarelativistic limit. Thus, the acceleration vanishes only when $v \rightarrow v_{\mathrm{cr}}$, where the critical velocity is determined by the equation $v G v=0$.
Let us estimate the critical velocity. Using the consequence $(\dot{x} S F \dot{x})=-b(\mu-1)(\dot{x} F S S F \dot{x})$ of the Pirani condition and the expression $S^{\mu}{ }_{\alpha} S^{\alpha \nu}=-4\left[\pi^{2} \omega^{\mu} \omega^{\nu}+\omega^{2} \pi^{\mu} \pi^{\nu}\right]$, we write

$$
\begin{aligned}
& -(\dot{x} G \dot{x}) \\
& =c^{2}-\mathbf{v}^{2} \\
& \quad+4 b^{2}(\mu-1)^{2}\left[\pi^{2}(\omega F \dot{x})^{2}+\omega^{2}(\pi F \dot{x})^{2}\right] .
\end{aligned}
$$

As $\pi$ and $\omega$ are space-like vectors (see the discussion below (70)), the last term is nonnegative, so $v_{\mathrm{cr}} \geq c$. We show that generally this term is nonvanishing function of velocity; then $v_{\mathrm{cr}}>c$. Assume the contrary that this term vanishes at some velocity; then

$$
\begin{aligned}
& \omega F \dot{x}=-\omega^{0}(\mathbf{E v})+(\boldsymbol{\omega}, c \mathbf{E}+\mathbf{v} \times \mathbf{B})=0, \\
& \pi F \dot{x}=-\pi^{0}(\mathbf{E v})+(\pi, c \mathbf{E}+\mathbf{v} \times \mathbf{B})=0 .
\end{aligned}
$$

This implies $c(\mathbf{D E})+(\mathbf{D}, \mathbf{v} \times \mathbf{B})=0$. Consider the case $\mathbf{B}=$ 0 ; then it should be $(\mathbf{D E})=0$. On the other hand, for the homogeneous field, the quantity $S^{\mu \nu} F_{\mu \nu}=2[(\mathrm{DE})+2(\mathrm{SB})]=$ $2(\mathrm{DE})$ is a constant of motion. Hence, we can take the initial conditions for spin such that $(\mathbf{D E}) \neq 0$ at any instant; this implies $v_{\mathrm{cr}}>c$.

7.3. Ultrarelativistic Limit within the Geometry Determined by Effective Metric. According to the previous section, if we insist on preserving usual special-relativity definitions of time and distance (175), the speed of light does not represent special point of the equation for trajectory. Acceleration of the particle with anomalous magnetic moment generally vanishes at the speed slightly higher than the speed of light. Hence, we arrive at a rather surprising result that speed of light does not represent maximum velocity of manifestly relativistic equation (237). This state of affairs is unsatisfactory because the Lorentz transformations have no sense above $c$, so two observers with relative velocity $c<v<v_{\text {cr }}$ will not be able to compare results of their measurements.

To keep the condition $v_{\mathrm{cr}}=c$, we use formal similarity of the matrix $G$ which appeared in (223) with space-time metric. Then, we can follow the general-relativity prescription of Section 2 to define time and distance in the presence of electromagnetic field; that is, we use $G$ of (223) to define three-dimensional geometry (5)-(8). The effective metric depends on $x^{i}$ via the field strength $F\left(x^{0}, x^{i}\right)$ and on $x^{0}$ via the field strength as well as via the spin-tensor $S\left(x^{0}\right)$. So, the effective metric is time-dependent even in stationary electromagnetic field. With these definitions we have, by construction, $-\dot{x} G \dot{x}=\left(d t / d x^{0}\right)^{2}\left(c^{2}-(\mathbf{v} \gamma \mathbf{v})\right)$, so the critical speed coincides with the speed of light. The intervals of time and distance are given now by (5) and (6); they slightly differ from those in empty space. 
In the present case, the expression for three-acceleration can be obtained in closed form in an arbitrary electromagnetic field. We present (226) in the form in (44):

$$
\begin{aligned}
& D D x^{\mu}=\mathscr{F}^{\mu}=-D x^{\mu} \frac{D m_{r}(S)}{m_{r}}-T^{\mu}{ }_{\nu} D \widetilde{\mathscr{T}}_{\alpha}^{\nu}(S) D x^{\alpha} \\
& +T^{\mu}{ }_{\nu}\left\{\frac{e}{m_{r} c^{2}}(F D x)^{\nu}+\frac{\mu e}{4 m_{r}^{2} c^{3}} \partial^{\nu}(S F)\right. \\
& \left.+\frac{\mu}{m_{r}} D Z^{\nu}\right\} .
\end{aligned}
$$

Then, the acceleration is given by (46). The first two terms on right hand side of (244) give potentially divergent contributions arising from the piece $\dot{S} \sim 1 / \sqrt{c^{2}-\mathbf{v} \gamma \mathbf{v}}$ of (227). In the previous section, we have seen that the dangerous contribution contained in the second term disappears. To analyze the first term, we substitute $\mathscr{F}^{i}$ from (244) into (46). Using the property $\widetilde{M}^{i}{ }_{j} v^{j}=v^{i}\left(\left(c^{2}-\mathbf{v} \gamma \mathbf{v}\right) / c^{2}\right)$, we obtain the acceleration

$$
\begin{aligned}
a^{i} & =\left(c^{2}-\mathbf{v} \gamma \mathbf{v}\right)\left[-v^{i} \frac{\dot{m}_{r}}{m_{r} c^{2}}-\frac{\widetilde{M}_{j}^{i} T^{j}{ }_{\nu} \dot{\widetilde{T}}_{\alpha}^{\nu} v^{\alpha}}{c^{2}-\mathbf{v} \gamma \mathbf{v}}\right. \\
& +\widetilde{M}_{j}^{i}{ }_{j}{ }^{j}{ }_{\nu}\left\{\frac{e}{m_{r} c^{2} \sqrt{c^{2}-\mathbf{v} \gamma \mathbf{v}}(F v)^{\nu}}\right. \\
& \left.\left.+\frac{\mu e}{4 m_{r}^{2} c^{3}} \partial^{v}(S F)+\frac{\mu}{m_{r} \sqrt{c^{2}-\mathbf{v} \gamma \mathbf{v}}} \dot{Z}^{\nu}\right\}\right] \\
& +\widetilde{M}_{j}^{i} \widetilde{\Gamma}_{k l}^{j}(\gamma) v^{k} v^{l}+\frac{1}{2}\left(\frac{d t}{d x^{0}}\right)^{-1}\left[\left(\mathbf{v} \partial_{0} \gamma \gamma^{-1}\right)^{i}\right. \\
& \left.-\frac{v^{i}}{c^{2}}\left(\mathbf{v} \partial_{0} \gamma \mathbf{v}\right)\right],
\end{aligned}
$$

so the divergency due to $\dot{m}_{r} \sim 1 / \sqrt{c^{2}-\mathbf{v} \gamma \mathbf{v}}$ is cancelled by the factor in front of this term. In the result, the acceleration is finite as $v \rightarrow c$. Besides, taking into account the property $(\mathbf{v} \gamma)_{i} \widetilde{M}_{j}^{i}=(\mathbf{v} \gamma)_{j}\left(\left(c^{2}-\mathbf{v} \gamma \mathbf{v}\right) / c^{2}\right)$, we conclude that longitudinal acceleration (47),

$$
\begin{aligned}
\mathbf{v} \gamma \mathbf{a} & =\frac{\left(c^{2}-\mathbf{v} \gamma \mathbf{v}\right)^{2}}{c^{2}}(\mathbf{v} \gamma \mathscr{F}) \\
& +\frac{c^{2}-\mathbf{v} \gamma \mathbf{v}}{c^{2}}\left[(\mathbf{v} \gamma)_{i} \widetilde{\Gamma}_{k l}^{i}(\gamma) v^{k} v^{l}\right. \\
& \left.+\frac{1}{2}\left(\frac{d t}{d x^{0}}\right)^{-1}\left(\mathbf{v} \partial_{0} \gamma \mathbf{v}\right)\right]
\end{aligned}
$$

vanishes in this limit.

\section{Conclusion}

In this work we have studied behavior of ultrarelativistic spinning particle in external fields. To construct interaction of spin with external fields and to analyze its influence on the trajectory of the particle, we used the vector model of spin. Minimal interaction with gravity was formulated starting from the Lagrangian variational problem without auxiliary variables (56). The nonminimal interaction with gravity through the gravimagnetic moment $\kappa$ [25] has been achieved in the Lagrangian with one auxiliary variable (170).

The variational problems imply the fixed value of spin (78); that is, they correspond to an elementary spin onehalf particle. The vector model also allowed us to construct Lagrangian action (94) with unfixed spin and with a massspin trajectory constraint, that is, with the properties of Hanson-Regge relativistic top [22]. In this model appeared the fundamental length scale and spin has four physical degrees of freedom. At last, interaction of spinning particle with magnetic moment $\mu$ with an arbitrary electromagnetic field was achieved in the Lagrangian action with one auxiliary variable (204). Equations of motion of minimally interacting spinning particle (i.e., with $\kappa=0$ ) have been identified with Mathisson-Papapetrou-Tulczyjew-Dixon equations. They are widely used in the current literature for description of rotating bodies in general relativity. To study the class of trajectories of a body with fixed integrals of motion $\sqrt{-P^{2}}=k$ and $S^{2}=\beta$, we can use our spinning particle with $m=k / c$ and $\alpha=\beta / 8$.

To study our general-covariant equations in the laboratory frame, we used the Landau-Lifshitz $1+3$-splitting formalism of four-dimensional pseudo Riemann space, where the basic structure is a congruence of one-dimensional timelike curves identified with world lines of the laboratory clocks. This formalism allows one to determine the time interval, distance, and then velocity between two infinitesimally closed points $x^{\mu}$ and $x^{\mu}+\delta x^{\mu}$ of the particle's world line. The basic requirement for definition of the three-dimensional quantities is that speed of light should be a coordinateindependent notion. Due to the decomposition of space time into time + space, one manipulates only time-varying vector and tensor fields. In the resulting three-dimensional geometry with Riemannian scalar product, we asked about the notion of a constant vector field. We have suggested notion (27) which follows from the geometric requirement that scalar product of constant fields does not depend on the point where it was computed. For the vector field of velocity, its deviation from the constant field has given us acceleration (28). Then, we showed that the definition adopted is consistent with the basic principle of general relativity: massive spinless particle, propagating in a gravitational field along a four-dimensional geodesic, can not exceed the speed of light. With this definition at hand, we analyzed ultrarelativistic behavior of the spinning particle in external fields.

Evolution of the fast MPTD particle in the laboratory frame was studied on the base of Lagrangian equations (92) and (93). In these equations, we observed the emergence of effective metric (89) instead of the original one. We have examined the two metrics as candidates for construction of 
three-dimensional space-time geometry (5)-(8) probed by the particle. In both cases, the MPTD equations have unsatisfactory behavior in the ultrarelativistic limit. In particular, three-dimensional acceleration (28) increases with velocity and becomes infinite in the limit.

Further, we showed that spinning particle with $\kappa=1$ is free of the problems detected in MPTD equations. For this value of gravimagnetic moment, the effective metric does not appear and the three-dimensional geometry should be defined, unambiguously, with respect to the original metric. Critical velocity of the theory coincides with the speed of light and three-dimensional acceleration vanishes as $v \rightarrow c$. So, the spinning particle with gravimagnetic moment $\kappa=1$ seems a more promising candidate for the description of a relativistic rotating body in general relativity. An interesting property of the resulting equations is that spin ceases to affect the trajectory in ultrarelativistic limit; the trajectory of spinning particle becomes more and more close to that of spinless particle as $v \rightarrow c$. Besides, the spin precesses with finite angular velocity in this limit.

Equations in electromagnetic and in gravitational fields become very similar after the identification of $\mu \sim \kappa$ and $R_{\mu \nu \alpha \beta^{\alpha \beta}} \sim F_{\mu \nu}$. In particular, interaction of spin with electromagnetic field in Minkowski space also produces effective metric (223) for the particle with anomalous magnetic moment $\mu \neq 1$. If we insist on the usual special-relativity notions of time and distance, the critical speed turns out to be more than the speed of light. To preserve the equality $v_{\mathrm{cr}}=$ $c$, we are forced to assume that particle in electromagnetic field probes the three-dimensional geometry determined with respect to the effective metric instead of the Minkowski metric. In the result, we have rather unusual picture of the Universe filled with spinning matter. Since $G$ depends on spin, in this picture there is no unique space-time manifold for the Universe of spinning particles; each particle will probe its own three-dimensional geometry. In principle, this could be an observable effect. With effective metric (223), (5) implies that the time of life of muon in electromagnetic field and in empty space should be different.

\section{Competing Interests}

The authors declare that they have no competing interests.

\section{Acknowledgments}

The work of Alexei A. Deriglazov has been supported by the Brazilian foundations CNPq (Conselho Nacional de Desenvolvimento Científico e Tecnológico, Brazil) and FAPEMIG (Fundacão de Amparo á Pesquisa do Estado de Minas Gerais, Brazil). Walberto Guzmán Ramírez thanks CAPES for the financial support (Program PNPD/2011).

\section{References}

[1] A. Trautman, "Lectures on general relativity," General Relativity and Gravitation, vol. 34, no. 5, pp. 715-762, 2002.

[2] M. Mathisson, "Neue Mechanik materieller Systeme," Acta Physica Polonica B, vol. 6, pp. 163-2900, 1937, Republication:
General Relativity and Gravitation, vol. 42, no. 4, pp. 1011-1048, 2010.

[3] A. Papapetrou, "Spinning test-particles in general relativity I," Proceedings of the Royal Society. London. Series A. Mathematical, Physical and Engineering Sciences, vol. 209, pp. 248-258, 1951.

[4] W. M. Tulczyjew, "Motion of multipole particles in general relativity theory binaries," Acta Physica Polonica, vol. 18, p. 393, 1959.

[5] W. G. Dixon, "A covariant multipole formalism for extended test bodies in general relativity," Il Nuovo Cimento, vol. 34, pp. 317339, 1964.

[6] W. G. Dixon, "Classical theory of charged particles with spin and the classical limit of the Dirac equation," Il Nuovo Cimento, vol. 38, pp. 1616-1643, 1965.

[7] F. A. E. Pirani, "On the physical significance of the Riemann tensor," Acta Physica Polonica. B, vol. 15, pp. 389-405, 1956.

[8] L. H. Thomas, "The kinematics of an electron with an axis," Philosophical Magazine and Journal of Science, vol. 3 S.7, no. 13, p. 1, 1927.

[9] J. Frenkel, "Die elektrodynamik des rotierenden elektrons," Zeitschrift für Physik, vol. 37, no. 4-5, pp. 243-262, 1926.

[10] J. Frenkel, "Spinning electrons," Nature, vol. 117, no. 2949, pp. 653-654, 1926.

[11] V. Bargmann, L. Michel, and V. L. Telegdi, "Precession of the polarization of particles moving in a homogeneous electromagnetic field," Physical Review Letters, vol. 2, no. 10, pp. 435-436, 1959.

[12] A. O. Barut, Electrodynamics and Classical Theory of Fields and Particles, MacMillan, New York, NY, USA, 1964.

[13] H. C. Corben, Classical and Quantum Theories of Spinning Particles, Holden-Day, San Francisco, Calif, USA, 1968.

[14] A. A. Pomeranskii and I. B. Khriplovich, "Equations of motion of a spinning relativistic particle in external fields," Journal of Experimental and Theoretical Physics, vol. 86, no. 5, pp. 839-849, 1998, Translated from Zhurnal Eksperimental'noi $i$ Teoreticheskoi Fiziki, vol. 113, pp. 1537-1557, 1998.

[15] C. M. Will, "The confrontation between general relativity and experiment," Living Reviews in Relativity, vol. 17, article 4, 2014.

[16] A. B. Balakin and V. A. Popov, "Spin-axion coupling," Physical Review D, vol. 92, no. 10, Article ID 105025, 16 pages, 2015.

[17] A. A. Deriglazov and A. M. Pupasov-Maksimov, "Lagrangian for Frenkel electron and position's non-commutativity due to spin," European Physical Journal C, vol. 74, no. 10, article 3101, 2014.

[18] A. M. Frydryszak, "Lagrangian models of the particles with spin: the first seventy years," https://arxiv.org/abs/hep-th/ 9601020.

[19] D. Mukunda, H. van Dam, and L. C. Biedenharn, Relativistic Models of Extended Hadrons Obeying a Mass-Spin Trajectory Constraint, vol. 165 of Lectures in Mathematical Physics, Springer, Berlin, Germany, 1982.

[20] A. A. Deriglazov and W. G. Ramírez, "Mathisson-PapapetrouTulczyjew-Dixon (MPTD) equations in ultra-relativistic regime and gravimagnetic moment," http://arxiv.org/abs/1509.05357.

[21] A. A. Deriglazov and W. G. Ramirez, "World-line geometry probed by fast spinning particle," Modern Physics Letters A. Particles and Fields, Gravitation, Cosmology, Nuclear Physics, vol. 30, no. 21, Article ID 1550101, 2015.

[22] A. J. Hanson and T. Regge, "The relativistic spherical top," Annals of Physics, vol. 87, no. 2, pp. 498-566, 1974. 
[23] S. Hojman, "Lagrangian theory of motion of spinning particles in torsion gravitational theories," Physical Review D, vol. 18, no. 8, pp. 2741-2744, 1978.

[24] C. Armaza, M. Bañados, and B. Koch, "Can Schwarzschild black holes be accelerators of spinning massive particles?" http://arxiv .org/abs/1510.01223.

[25] I. B. Khriplovich, "Particle with internal angular momentum in a gravitational field," Journal of Experimental and Theoretical Physics, vol. 69, no. 2, p. 217, 1989, Translated from: Zhurnal Eksperimental'noi i Teoreticheskoi Fiziki, vol. 96, pp. 385, 1989.

[26] J. W. van Holten, "On the electrodynamics of spinning particles," Nuclear Physics B, vol. 356, no. 1, pp. 3-26, 1991.

[27] L. D. Landau and E. M. Lifshitz, The Classical Theory of Fields, vol. 2 of Course of Theoretical Physics, Pergamon Press, 3rd edition, 2011.

[28] E. Gourgoulhon, "3+1 Formalism in general relativity," in Bases of Numerical Relativity, vol. 846 of Lecture Notes in Physics, p. 1, Springer, 2012.

[29] A. Deriglazov, Classical Mechanics: Hamiltonian and Lagrangian Formalism, Springer, Berlin, Germany, 2010.

[30] W. Guzmán Ramírez, A. A. Deriglazov, and A. M. PupasovMaksimov, "Frenkel electron and a spinning body in a curved background," Journal of High Energy Physics, vol. 2014, p. 109, 2014.

[31] A. A. Deriglazov and A. M. Pupasov-Maksimov, "Frenkel electron on an arbitrary electromagnetic background and magnetic Zitterbewegung," Nuclear Physics B, vol. 885, pp. 1-24, 2014.

[32] A. A. Deriglazov, "Lagrangian for the Frenkel electron," Physics Letters B, vol. 736, pp. 278-282, 2014.

[33] W. G. Ramirez and A. A. Deriglazov, "Lagrangian formulation for Mathisson-Papapetrou-Tulczyjew-Dixon equations," Physical Review D, vol. 92, no. 12, Article ID 124017, 2015.

[34] A. Staruszkiewicz, "Fundamental relativistic rotator," Acta Physica Polonica B, Proceedings Supplement, vol. 1, no. 1, pp. 109-112, 2008.

[35] A. A. Deriglazov and A. M. Pupasov-Maksimov, "Geometric constructions underlying relativistic description of spin on the base of non-Grassmann vector-like variable," Symmetry, Integrability and Geometry: Methods and Applications, vol. 10, article 012, 13 pages, 2014.

[36] P. A. M. Dirac, Lectures on Quantum Mechanics, Yeshiva University, New York, NY, USA, 1964.

[37] D. M. Gitman and I. V. Tyutin, Quantization of Fields with Constraints, Springer Series in Nuclear and Particle Physics, Springer, Berlin, Germany, 1990.

[38] K. S. Thorne and J. B. Hartle, "Laws of motion and precession for black holes and other bodies," Physical Review D, vol. 31, no. 8, pp. 1815-1837, 1985. 

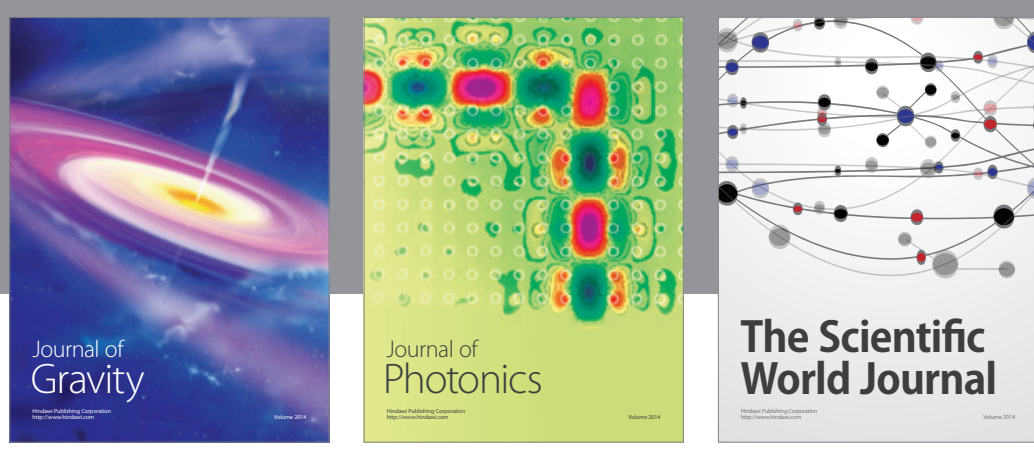

The Scientific World Journal
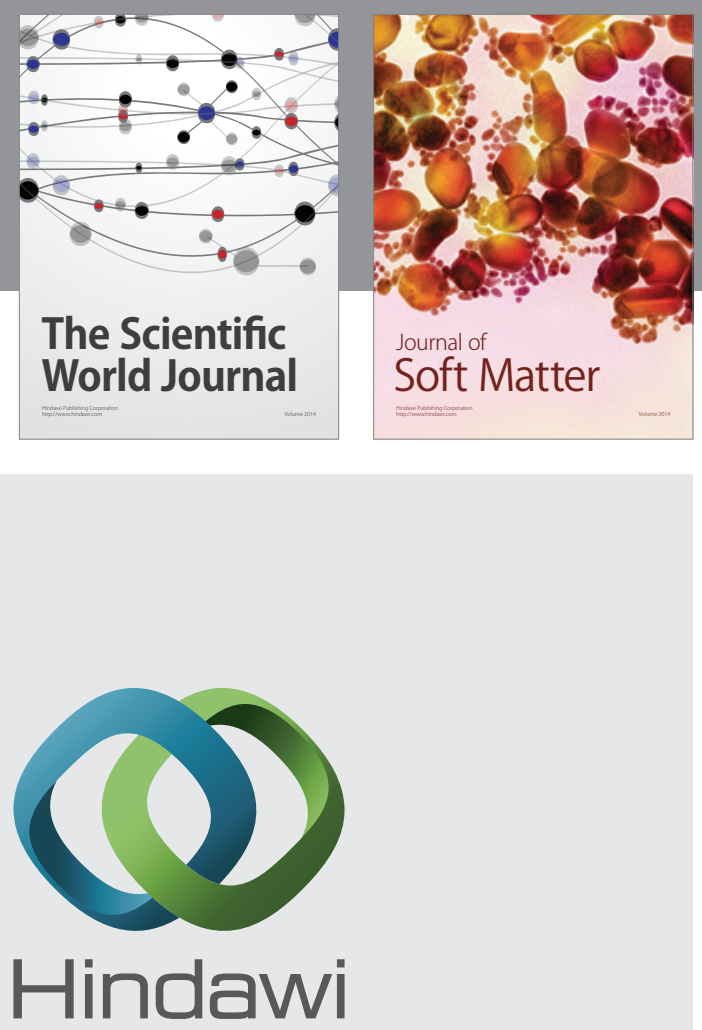

Submit your manuscripts at

http://www.hindawi.com

nternational Journal of

Statistical Mechanics
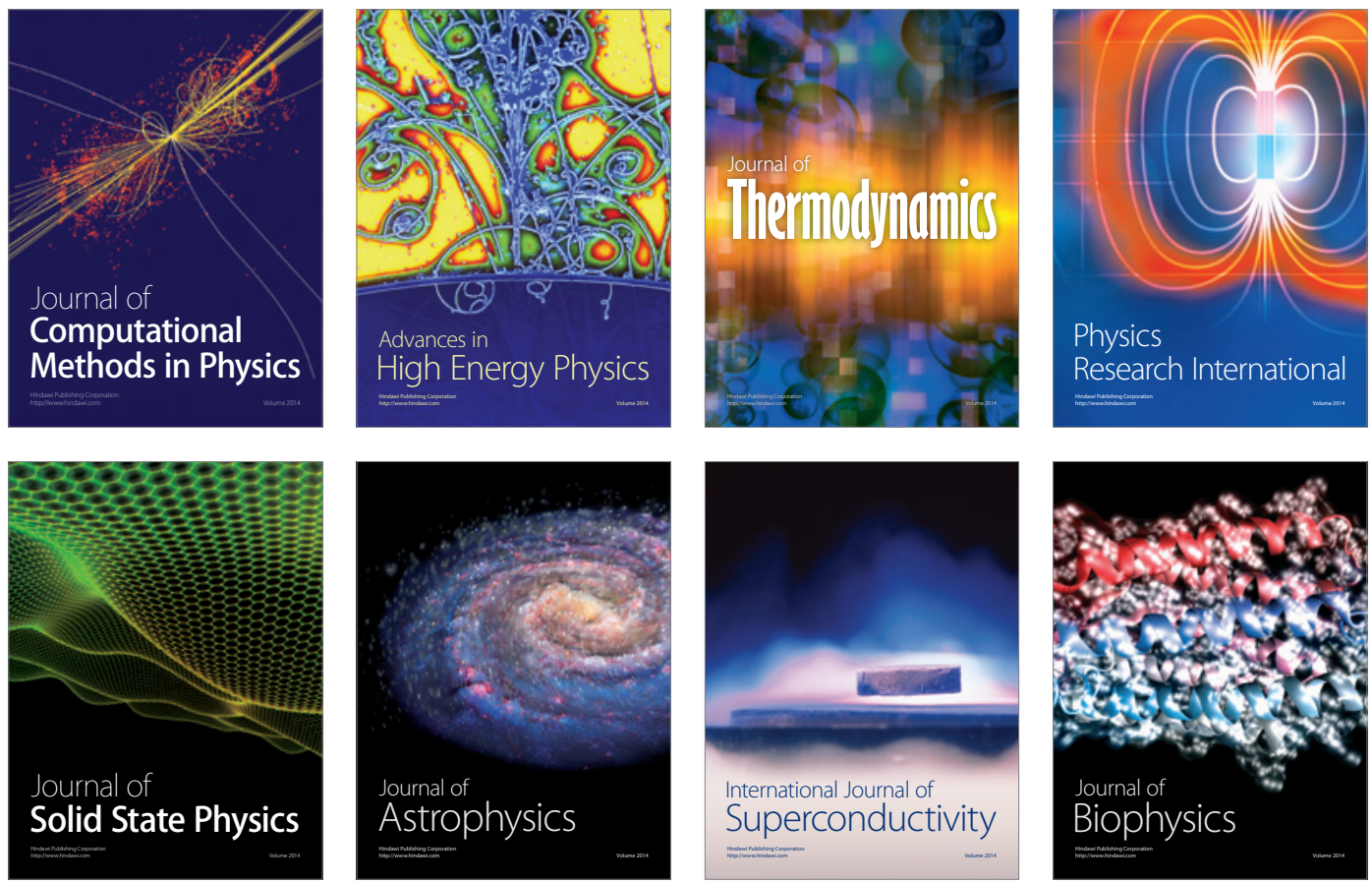
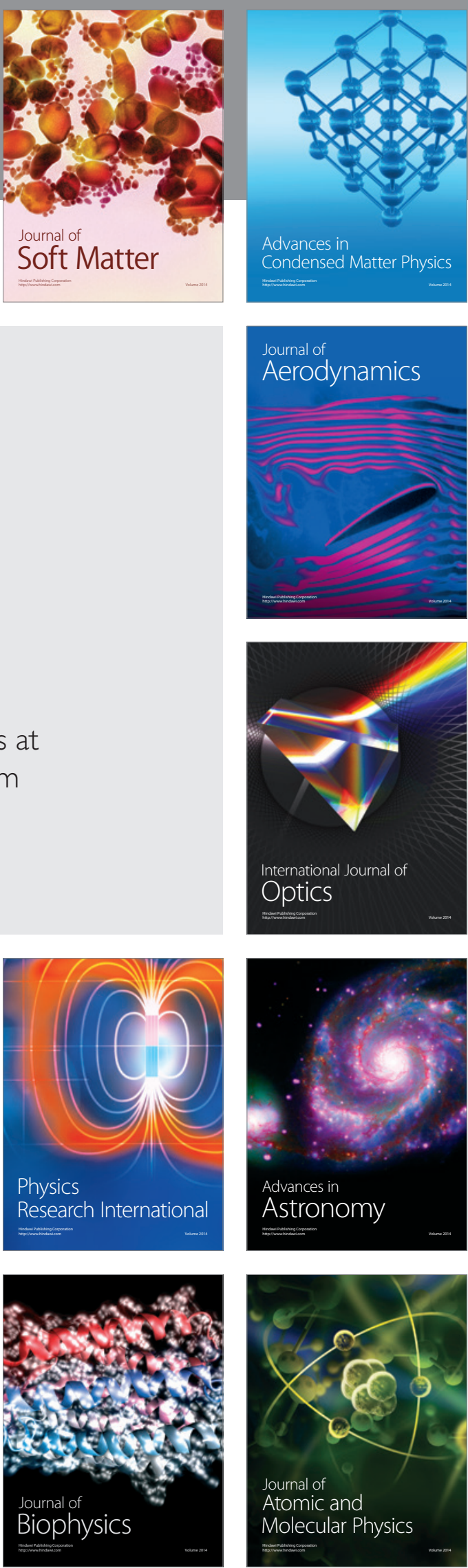